MATHEMATICS OF COMPUTATION

Volume 80, Number 274, April 2011, Pages 1163-1196

S 0025-5718(2010)02430-5

Article electronically published on October 28, 2010

\title{
AN EFFECTIVE BOUND FOR THE HUBER CONSTANT FOR COFINITE FUCHSIAN GROUPS
}

\author{
J. S. FRIEDMAN, J. JORGENSON, AND J. KRAMER
}

ABstract. Let $\Gamma$ be a cofinite Fuchsian group acting on hyperbolic two-space $\mathbb{H}$. Let $M=\Gamma \backslash \mathbb{H}$ be the corresponding quotient space. For $\gamma$, a closed geodesic of $M$, let $l(\gamma)$ denote its length. The prime geodesic counting function $\pi_{M}(u)$ is defined as the number of $\Gamma$-inconjugate, primitive, closed geodesics $\gamma$ such that $e^{l(\gamma)} \leq u$. The prime geodesic theorem states that:

$$
\pi_{M}(u)=\sum_{0 \leq \lambda_{M, j} \leq 1 / 4} \operatorname{li}\left(u^{s_{M, j}}\right)+O_{M}\left(\frac{u^{3 / 4}}{\log u}\right),
$$

where $0=\lambda_{M, 0}<\lambda_{M, 1}<\cdots$ are the eigenvalues of the hyperbolic Laplacian acting on the space of smooth functions on $M$ and $s_{M, j}=\frac{1}{2}+\sqrt{\frac{1}{4}-\lambda_{M, j}}$. Let $C_{M}$ be the smallest implied constant so that

$$
\left|\pi_{M}(u)-\sum_{0 \leq \lambda_{M, j} \leq 1 / 4} \operatorname{li}\left(u^{s_{M, j}}\right)\right| \leq C_{M} \frac{u^{3 / 4}}{\log u} \quad \text { for all } u>1 .
$$

We call the (absolute) constant $C_{M}$ the Huber constant.

The objective of this paper is to give an effectively computable upper bound of $C_{M}$ for an arbitrary cofinite Fuchsian group. As a corollary we bound the Huber constant for $\operatorname{PSL}(2, \mathbb{Z})$, showing that $C_{M} \leq 16,607,349,020,658 \approx$ $\exp (30.44086643)$.

\section{INTRODUCTION}

Let $\Gamma$ be a cofinite Fuchsian group, and let $M=\Gamma \backslash \mathbb{H}$ be the corresponding hyperbolic orbifold. Let $\mathcal{C}(M)$ denote the set of closed geodesics of $M$, and let $\mathcal{P}(M)$ denote the set of prime (or primitive) closed geodesics (see Bus92, page 245]). For each $\gamma \in \mathcal{C}(M)$ there exists a unique prime geodesic $\gamma_{0}$ and a unique exponent $m \geq 1$ so that $\gamma=\gamma_{0}^{m}$. Let $l(\gamma)$ denote the length of $\gamma$. Associated to $\gamma$ is a unique hyperbolic conjugacy class $\left\{P_{\gamma}\right\}_{\Gamma}$ with norm

$$
N_{\gamma} \equiv N\left(P_{\gamma}\right)=e^{l(\gamma)} .
$$

The prime geodesic counting function $\pi_{M}(u)$ is defined to be the number of $\Gamma$-inconjugate, primitive, hyperbolic elements $\gamma \in \Gamma$ such that $e^{l_{\gamma}}<u$.

Received by the editor September 25, 2009 and, in revised form, March 2, 2010.

2010 Mathematics Subject Classification. Primary 11F72; Secondary 30F35.

The second named author acknowledges support from grants from the NSF and PSC-CUNY..

The third named author acknowledges support from the DFG Graduate School Berlin Mathematical School and from the DFG Research Training Group Arithmetic and Geometry.

(C)2010 American Mathematical Society Reverts to public domain 28 years from publication 
Selberg [Sel56] and Huber Hub59, Hub61a, Hub61b] independently proved the prime geodesic theorem, which is the asymptotic formula

$$
\pi_{M}(u) \sim \frac{u}{\log u}, \quad(u \rightarrow \infty) .
$$

Later, Huber proved a stronger version of the prime geodesic theorem, with error term: 1 :

$$
\pi_{M}(u)=\sum_{0 \leq \lambda_{M, j} \leq 1 / 4} \operatorname{li}\left(u^{s_{M, j}}\right)+O_{M}\left(\frac{u^{3 / 4}}{\log u}\right),
$$

where $0=\lambda_{M, 0}<\lambda_{M, 1}<\cdots$ are the eigenvalues of the hyperbolic Laplacian acting on the space of smooth functions on $M$ and

$$
s_{M, j}=\frac{1}{2}+\sqrt{\frac{1}{4}-\lambda_{M, j}} .
$$

For a proof of this theorem, via the Selberg zeta function, see Hej76, Hej83 and Ran78. Randol Ran84 and Sarnak Sar80 also gave of proof using the Selberg trace formula. We call the implied constant inherent in $O_{M}\left(\frac{u^{3 / 4}}{\log u}\right)$ the Huber constant, denoted by $C_{M}$. The Huber constant is an absolute constant, not an asymptotic constant2. The Huber constant is the minimal constant that satisfies

$$
\left|\pi_{M}(u)-\sum_{0 \leq \lambda_{M, j} \leq .1 / 4} \operatorname{li}\left(u^{s_{M, j}}\right)\right| \leq C_{M}\left(\frac{u^{3 / 4}}{\log u}\right) \quad \text { for all } u>1 .
$$

The main goal of this paper is to estimate $C_{M}$ for an arbitrary cofinite Fuchsian group. We determine an effective algorithm by which one can obtain explicit bounds for $C_{M}$ using elementary geometric and spectral theoretic data associated to $M$. In particular, we apply the algorithm in the case of the full modular group $\operatorname{PSL}(2, \mathbb{Z})$ and obtain a precise, numerical bound for the Huber constant.

In JK02, the authors studied the Huber constant in the following situation: Let $\Gamma_{0}$ be a cofinite Fuchsian group, and let $\Gamma$ be a finite-index subgroup of $\Gamma_{0}$. They showed that

$$
C_{M} \leq\left[\Gamma_{0}: \Gamma\right] \cdot C_{M_{0}} .
$$

Hence, if one could estimate this constant for $\Gamma_{0}=\operatorname{PSL}(2, \mathbb{Z})$, one would have an estimate for any congruence subgroup. For more applications of these ideas, see [JK09, JK06, JK01.

In the articles [JK09, JK06 and JK01, the authors studied analytic aspects of Arakelov theory, ultimately developing bounds for special values of Selberg's zeta functions, Green's functions and Faltings's delta function. The bounds for these analytic functions involved many explicitly computable analytic and geometric quantities, together with the Huber constant. As such, specific, effective bounds for the Huber constant then could be used to make the bounds derived in JK09, JK06 and JK01 effective. With this said, the results in the present paper complete the analysis in the aforementioned articles, thus providing effective, numerically computable, bounds for various analytic quantities which appear in the Arakelov theory of algebraic curves.

\footnotetext{
${ }^{1}$ Huber's error term was slightly different: $O\left(u^{3 / 4}(\log u)^{-1 / 2}\right)$.

${ }^{2}$ If $C_{M}$ was an asymptotic constant, it would equal zero for the modular group since there are better estimates for the error term Iwa02.
} 
TABLE 1. Fundamental constants

\begin{tabular}{|c|c|}
\hline Constant & Value \\
\hline \hline$\Gamma$ & cofinite Fuchsian group with fundamental domain $\mathcal{F}$ \\
\hline$\tau$ & number of inequivalent parabolic cusps \\
\hline$Y$ & $\mathcal{F}=\mathcal{F}_{Y} \cup \bigcup_{i=0}^{\tau} \mathcal{F}_{i}^{Y}$, a decomposition of $\mathcal{F}$ into cusp sectors $\mathcal{F}_{i}^{Y}$ and compact set $\mathcal{F}_{Y}$ \\
\hline$\theta_{R}, m_{R}$ & $\operatorname{tr}(R)=2 \cos \left(\theta_{R}\right), 0<\theta_{R}<\pi ; m_{R}=|C(R)|$ \\
\hline$d$ & For cocompact $\Gamma d$ is the (hyperbolic) diameter of $\mathcal{F}$ \\
\hline $\mathcal{A}$ & number of eigenvalues $\lambda$ of $\Delta$, such that $\lambda \leq \frac{1}{4}$, including $\lambda_{0}=0$ \\
\hline$s_{1}$ & $\left\{\begin{array}{cc}\frac{1}{2}+\sqrt{\frac{1}{4}-\lambda_{1}} \quad \lambda_{1} \leq \frac{1}{4} \\
\infty\end{array} \quad \begin{array}{c}\lambda_{1}>\frac{1}{4}, \\
\hline \mathcal{N}\end{array}\right.$ \\
\hline$c_{1}$ & $\sigma_{1} \geq \cdots \geq \sigma_{\mathcal{N}}$ are all the poles of $\phi(s)$ in the interval $\left(\frac{1}{2}, 1\right]$ \\
\hline$c$ & minimum of the lower left hand matrix entry of non-parabolic elements \\
\hline$\mu$ & chosen to satisfy $1<c<N(\Gamma)$ for all hyperbolic $\gamma \in \Gamma$ \\
\hline
\end{tabular}

Main result. Our main result is Theorem 4.15 (see \$4.8), where we determine an upper bound for the Huber constant $C_{M}$ in terms of various well studied invariants of $\Gamma$ and $M$ such as: the number of small eigenvalues $\mathcal{A}$, the number of exceptional poles of the scattering matrix $\mathcal{N}$, the smallest positive eigenvalue $\lambda_{1}$, the length of the smallest closed geodesic of $M$, the decomposition of the fundamental domain of $\Gamma$ into cusp sectors, the area of $M$, and a few other simple, easily computable, invariant 3 . We could give the main result by stating our upper bound for $C_{M}$. However, the result is so complicated that it would take two pages just to list out the equation. So, we present our results as an algorithm, rather than a formula. To help the reader appreciate the complexity (for the case of a general cofinite Fuchsian group), we state, as a corollary, the simple case of a cocompact, torsionfree Fuchsian group.

In the process of proving Theorem 4.15, we give an upper bound for the implied (absolute) constant of the spectral counting $\mu(r)$; see Theorem 3.3. In other words, for an arbitrary cofinite Fuchsian group, we find an explicit constant $C$, depending on $\Gamma$, so that

$$
\mu(r) \equiv\left|\left\{r_{n} \mid 0 \leq r_{n} \leq r\right\}\right|+\frac{1}{4 \pi} \int_{-r}^{r}\left|\frac{\phi^{\prime}}{\phi}\left(\frac{1}{2}+i t\right)\right| d t \leq C\left(r^{2}+\frac{1}{4}\right) \quad(r \geq 0) .
$$

Next, consider the following table of constants, which are valid only for $\Gamma$ cocompact, torsion-free.

Theorem. Let $\Gamma$ be a cocompact, torsion-free Fuchsian group. Then the constant $C_{u}$ is an upper bound for the Huber constant $C_{M}$, and the constant $C$ is an upper bound for the implied constant of the spectral counting function of $\Delta$.

In order to estimate $C_{M}$ for general cofinite Fuchsian group we need the following modified table. Some constants from the cocompact table are redefined.

Theorem. Let $\Gamma$ be a cofinite Fuchsian group. Then the constant $C_{u}$ is an upper bound for the Huber constant $C_{M}$, and the constant $C$ is an upper bound for the implied constant of the spectral counting function of $\Delta$.

\footnotetext{
${ }^{3}$ These invariants are all known or easily estimated for the modular group.
} 
TABLE 2. Algorithm for cocompact, torsion-free case

\begin{tabular}{|c|c|}
\hline Constant & Value \\
\hline \hline$C_{1}$ & $2 e-2, e=2.718 \ldots$ \\
\hline$B$ & $\frac{2 \pi e^{d}}{|\mathcal{F}|}(d$ is the diameter of $\mathcal{F})$ \\
\hline$C$ & $3\left(\frac{|\mathcal{F}|}{4 \pi}+745 B\right)$ \\
\hline $\mathcal{A}$ & number of small eigenvalues of $\Delta$ including $\lambda_{0}$ \\
\hline$C_{10}$ & $8480 \sqrt{\frac{e}{2 \pi}}$ \\
\hline$C_{12}$ & $(\mathcal{A}-1)\left(1+3 C_{1}+\frac{2}{1-s_{1}}\left(1+C_{1}\right)\right)+2 C_{1}+2$ \\
\hline$C_{13}$ & $\frac{41}{6} C \cdot C_{10}$ \\
\hline$C_{16}$ & $C_{12}+C_{13}+\frac{3}{2 \pi}|\mathcal{F}| C_{10}$ \\
\hline$C_{17}$ & $4 \mathcal{A}+4 C_{16}$ \\
\hline$C_{18}$ & $4 \mathcal{A}+5 C_{16}$ \\
\hline$c$ & $1<c<N(P)$ for all $P \in \Gamma, \operatorname{hyperbolic}$ \\
\hline$\mu$ & $C_{18}+\frac{8 \mathcal{A}+4 C_{18}}{1-1 / c}$ \\
\hline$C_{19}$ & $C_{19}+\frac{8 \mathcal{A}+4 C_{18}}{\mu}$ \\
\hline$C_{20}$ & $|c-2| \frac{1}{\log 2}+|2-\sqrt{c}| \frac{2}{\log c}$ \\
\hline$C_{21}$ & $\frac{1}{1-1 / \log (2)}$ \\
\hline$C_{22}$ & \\
\hline$C_{u}$ & $C_{21} \mathcal{A}+C_{20} \frac{c^{3 / 4} \log c}{+C_{20}+C_{20} C_{22}+\frac{3}{4} C_{20} C_{21}}$ \\
\hline
\end{tabular}

To show the usefulness of Theorem 4.15 and Theorem 3.3, we estimate their respective constants $\left(C\right.$ and $\left.C_{M}\right)$ for the modular group $\operatorname{PSL}(2, \mathbb{Z})$. The results are Theorem 3.4 and Theorem 4.16.

Theorem. Let $\Gamma=\operatorname{PSL}(2, \mathbb{Z})$. Let $\mu(r)$ be the spectral counting function (3.7), and let

Then for $r \geq 0$,

$$
C=1,682,997 \text {. }
$$

$$
\mu(r) \leq C\left(r^{2}+\frac{1}{4}\right) .
$$

Theorem. Let $\Gamma=\operatorname{PSL}(2, \mathbb{Z})$. Then $C_{M} \leq 16,607,349,020,658$.

Using the theory laid out in Sar82, and the excellent open-source computational program PARI/GP The05, we can explicitly calculate (and list out) the length spectrum for $\operatorname{PSL}(2, \mathbb{Z})$. Our computations suggest that

$$
C_{M}<2 .
$$

So it seems that our result is not sharp.

Why is our result for $\operatorname{PSL}(2, \mathbb{Z})$ so much larger than the experimental result? There are many reasons. Our bound for the spectral counting function is probably significantly larger when compared to Weyl's law, an asymptotic result. We are 
TABLE 3. Algorithm for the general cofinite Fuchsian case

\begin{tabular}{|c|c|}
\hline Constant & Value \\
\hline$C_{1}$ & $2 e-2$ \\
\hline$B$ & $4 \pi Y(Y+1) \sum_{j=1}^{\min (2, \tau)} e^{2 d_{j}}$, see Lemma 1.2 for more details \\
\hline$C_{3}$ & $8 \tau$ \\
\hline$C_{4}$ & $5 \sum_{\{R\}} \frac{1}{2 m_{R} \sin \theta_{R}}\left(\frac{1}{2 \theta_{R}}+\frac{1}{2\left(\pi-\theta_{R}\right)}\right)$ \\
\hline$C_{5}$ & $8 \tau+C_{4}+\frac{|\mathcal{F}|}{4 \pi}+745 B$ \\
\hline$C_{6}$ & $C_{5}+\left(2\left|\log c_{1}\right|+\frac{\mathcal{N}}{\left(\sigma_{\mathcal{N}}-\frac{1}{2}\right)^{2}}\right) \frac{\sqrt{5}}{\sqrt{16 \pi}}$ \\
\hline$C_{7}$ & $\frac{8}{4 \pi}\left(2\left|\log c_{1}\right|+\frac{\mathcal{N}}{\left(\sigma_{\mathcal{N}}-\frac{1}{2}\right)^{2}}\right)$ \\
\hline$C$ & $3 C_{6}+C_{7}$ \\
\hline $\mathcal{A}$ & number of small eigenvalues of $\Delta$ including $\lambda_{0}$ \\
\hline$C_{10}$ & $8480 \sqrt{\frac{e}{2 \pi}}$ \\
\hline$C_{12}$ & $(\mathcal{A}-1)\left(1+3 C_{1}+\frac{2}{1-s_{1}}\left(1+C_{1}\right)\right)+2 C_{1}+2$ \\
\hline$C_{13}$ & $\frac{41}{6} C \cdot C_{10}$ \\
\hline$C_{14}$ & $C_{10} \frac{296 \tau}{3 \pi}+C_{10} \frac{\tau}{2}+2 \tau \log 2$ \\
\hline$C_{15}$ & \begin{tabular}{c|l|}
$\frac{56 C_{10}}{3}$ & $\sum_{\{R\}} \frac{1}{2 m_{R} \sin \theta_{R}}$ \\
\end{tabular} \\
\hline$C_{16}$ & $C_{12}+C_{13}+C_{14}+C_{15}+\frac{3}{2 \pi}|\mathcal{F}| C_{10}$ \\
\hline$C_{17}$ & $4 \mathcal{A}+4 C_{16}$ \\
\hline$C_{18}$ & $4 \mathcal{A}+5 C_{16}$ \\
\hline$c$ & $1<c<N(P)$ for all $P \in \Gamma$, hyperbolic \\
\hline$\mu$ & $\log c$ \\
\hline$C_{19}$ & $C_{18}+\frac{8 \mathcal{A}+4 C_{18}}{1-1 / c}$ \\
\hline$C_{20}$ & $C_{19}+\frac{8 \mathcal{A}+4 C_{18}}{\mu}$ \\
\hline$C_{21}$ & $|c-2| \frac{1}{\log 2}+|2-\sqrt{c}| \frac{2}{\log c}$ \\
\hline$C_{22}$ & $\frac{1}{1-1 / \log (2)}$ \\
\hline$C_{u}$ & $C_{21} \mathcal{A}+C_{20} \frac{c^{3 / 4}}{\log c}+C_{20}+C_{20} C_{22}+\frac{3}{4} C_{20} C_{21}$ \\
\hline
\end{tabular}

mostly interested in how the Huber constant grows as a function of the various fundamental constants. Estimating the spectral counting function required absolute estimates of various infinite sums over primitive hyperbolic classes, and we used trivial bounds for the hyperbolic counting function. The non-trivial absolute bound being the sole objective of this paper. However, using $\operatorname{PSL}(2, \mathbb{Z})$ specific bounds on the spectral counting function, the result could be markedly improved.

Connection to existing literature. As stated above, the bound for the Huber constant obtained in the present paper allows one to complete the analysis in [JK09, JK06 and JK01, resulting in explicit and computable bounds for certain analytic 
quantities in Arakelov theory. We refer the interested reader to these articles for the precise statements of the main results, from which one can immediately employ the bounds obtained here in order to further strengthen the analysis presented in JK09, JK06] and [JK01. We will discuss further applicability of the present paper to other computational problems.

In ECJMB, the authors used ideas from Arakelov theory and developed a strategy to compute Galois representations modulo $\ell$ associated to a fixed modular form of arbitrary weight. Ultimately, the goal of ECJMB is to devise an algorithm, which has complexity that is polynomial in $\ell$. A summary of the ideas from ECJMB is given in Edi06, where the purpose is to focus attention to the weight twelve modular form associated to $\operatorname{PSL}(2, \mathbf{Z})$. The ideas from ECJMB have been used to achieve advances in many other computation problems; see, for example, Bo07, CL09, Cou09, and [La06 for specific results, as well as the survey article Chl08 for more general comments.

As stated in [ECJMB, a key component of the algorithm was to determine bounds for Arakelov Green's functions. F. Merkl provided sufficient bounds to complete the algorithm; however, the bounds from [JK06 are sharper and, as a result, improve the efficiency of the algorithm from [ECJMB. Theorem 4.8 of JK06 summarizes a bound for the Arakelov Green's function in terms of hyperbolic data, including a special value of the Selberg zeta function. This special value was studied in JK09 and now can be bounded explicitly using the bounds for the Huber constant developed in the present paper. Thus, the bounds obtained for the Huber constant proved here ultimately can be used in the algorithms constructed in ECJMB] and subsequent articles.

Going forward, it may be possible that the algorithms of [ECJMB], or similarly developed ideas, could yield further applications, in which case effective bounds for the analytic aspects of Arakelov theory would play a role in the implementation of the resulting algorithms. Indeed, there has been recent progress in expanding Arakelov theory to allow for singular, or non-compact metrics (see BKK05, BKK07, FG09, and Ha09). As a result, one can anticipate further computational algorithms which utilize these recent extensions of Arakelov theory, in which case the bounds from [JK09, JK06 and JK01 and the present paper may play a role.

Outline. In brief, the present paper amounts to a careful analysis of the proof of the prime geodesic theorem, making sure that each step in the proof yields explicitly computable constants. However straightforward the methodology may sound, there are many details which involve considerable analytic difficulties.

In $\$ 1$ we prove some useful lemmas and develop necessary notation. Our argument consists of many estimates, each with an implicit constant, and our notation helps to keep track of these constants. At the heart of our argument is a variant of Karamata's Tauberian Theorem (Lemma 1.1 in 1.2 that gives an absolute, instead of an asymptotic, result.

In $\$ 2$ we study the heat kernel $e^{-\Delta t}$ of the Laplace-Beltrami operator for the hyperbolic orbifold $M=\Gamma \backslash \mathbb{H}$. Our main tool is the Selberg trace formula. We specifically show that $e^{-\Delta t}=O(1 / t)$ for $t \in(0,5]$, and find the corresponding explicit $O$-constant. Note that we focus on the interval $(0,5]$ because small eigenvalues $(\lambda<1 / 4)$ are treated differently in many applications of the Selberg trace 
formula. We will estimate the spectral counting function $\mu(x)$ for $\lambda \geq 1 / 5$ giving us a small margin of error from the $1 / 4$ cutoff.

Next, in 93 , using results from $\$ 2$ and our variant of Karamata's Tauberian theorem (Lemma 1.1), we prove Theorem 3.3 which provides an effective bound for the spectral counting function. In 33.2 we specialize Theorem 3.3 to the case of $\Gamma=\operatorname{PSL}(2, \mathbb{Z})$.

Finally, in 4 using Theorem 3.3 , we obtain an upper bound for the Huber constant (Theorem 4.15) and in 4.9, we specialize to the case of $\Gamma=\operatorname{PSL}(2, \mathbb{Z})$.

Concluding remarks. We conclude the introduction by addressing a number of points which naturally arise when studying the computations presented in this article.

First, it is possible to further contract the algorithm for bounding the Huber constant and obtain a bound which can be related to the geometry of $M$. For example, the constant $c_{1}$ is the radius of the largest isometric circle in a certain fundamental domain for $M$; see page 38 of Iwa97. Also, the number of small eigenvalues can be bounded by the topology of $M$; see Zo82. However, to employ general results would only further worsen the bound for $C_{M}$ for special important cases. For example, for the congruence subgroups, the Selberg $1 / 4$ conjecture asserts there is only one small eigenvalue, whereas the bound from [ Zo82 implies that the number of small eigenvalues grows at most like the genus of the surface, which may be true in general cases but most likely is not true in special cases of particular importance.

Second, the heat kernel style of test functions which we analyze could be replaced by studying a different approach to the prime geodesic theorem, namely by finite difference methods applied to the test functions denoted by $\psi_{k}$; see Hej83. In our investigation, we saw no significant difference when looking ahead to the problem of bounding $C_{M}$, so we simply selected one approach. We will leave the problem of studying other ways of proving the prime geodesic theorem to see if the methodologies lead to improved bounds for the Huber constant.

Finally, it is somewhat striking to see that in the case of the full modular group the bound we prove is approximately $\exp (30.45)$ whereas the numerical investigations lead one to believe that the true bound is 2 , which differ by an order of $10^{14}$. In Fall 2003, the second named author (J.J.) was giving a talk at the Courant Institute at NYU on preliminary results associated to [JK01. (As described in ECJMB, it was then that Peter Sarnak communicated to the second named author (J.J.) the problem of bounding Arakelov Green's functions.) During the lecture, when the results of JK09 were described, Sarnak stated that he felt it would be difficult to obtain a precise, numerical bound for the Huber constant for any group, including the full modular group. With this stated, the present paper successfully addresses the problem of determining precise, effective bounds for $C_{M}$, and it remains to be seen how, or rather if, methods can be developed to refine the bound for the full modular group.

\section{Preliminary lemmas}

In this section we group together certain lemmas that will be used throughout this paper. Our key lemma is a variant of Karamata's Tauberian Theorem. 
1.1. Big ' $\mathrm{O}$ ' notation. Let $i \in \mathbb{N}$, and let $f, g$ be functions with a common domain $D$. The statement

$$
f(x)=\mathcal{O}_{i}(g(x))
$$

means that there exists a positive constant $C_{i}$ so that

$$
|f(x)| \leq C_{i}|g(x)| \quad \forall x \in D .
$$

If the domain in question is not clear, we will explicitly state it. We call the constant $C_{i}$ absolute 4 .

1.2. Karamata's Tauberian Theorem. In this section we prove a variation of Karamata's Tauberian Theorem [Wid41, pp. 189-192]. Our version keeps track of the implied constants in the original theorem.

We will apply the lemma below with the parameter $d=5$. Small eigenvalues $(\lambda<1 / 4)$ are treated differently in many applications of the Selberg trace formula. We will estimate the spectral counting function $\mu(x)$ for $\lambda \geq 1 / 5$ giving us a small margin of error from the $1 / 4$ cutoff.

Lemma 1.1. Let $\alpha(t)$ be a non-negative, non-decreasing function, continuous on all but a possibly countable subset of $(0, \infty)$. Suppose that $\alpha(0)=0$ and that the integral

$$
f(s) \equiv \int_{0}^{\infty} e^{-s t} d \alpha(t)
$$

converges for all $s>0$. Suppose further that for $C>0, d>0$,

$$
f(s) \leq \frac{C}{s}, \quad \text { for all } 0<s \leq d
$$

Then

$$
\alpha(t) \leq 3 C t \quad \text { for all } t \geq \frac{1}{d}
$$

Proof. For $x \in[0,1]$ let

$$
g(x)= \begin{cases}0 & 0 \leq x \leq e^{-1} \\ \frac{1}{x} & e^{-1} \leq x \leq 1\end{cases}
$$

Note that

$$
g(x) \leq 3 \quad(0 \leq x \leq 1) .
$$

Since $\alpha(t)$ is non-decreasing, we have, for $s>0$,

Next, observing that

$$
\int_{0}^{\infty} e^{-s t} g\left(e^{-s t}\right) d \alpha(t) \leq \int_{0}^{\infty} e^{-s t} 3 d \alpha(t)
$$

$$
g\left(e^{-w}\right)=\left\{\begin{array}{cc}
e^{w} & 0 \leq w \leq 1 \\
0 & 1<w
\end{array}\right.
$$

an elementary calculation using the fact that $\alpha(0)=0$ shows that

$$
\begin{aligned}
\int_{0}^{\infty} & e^{-s t} g\left(e^{-s t}\right) d \alpha(t)=\int_{0}^{1 / s} e^{-s t} g\left(e^{-s t}\right) d \alpha(t) \\
& =\int_{0}^{1 / s} e^{-s t} e^{s t} d \alpha(t)=\alpha(1 / s)-\alpha(0)=\alpha(1 / s) .
\end{aligned}
$$

\footnotetext{
${ }^{4}$ As opposed to an asymptotic constant, say $A_{i}$, that would satisfy $|f(x)| \leq A_{i}|g(x)|$ for $x$ sufficiently large.
} 
Hence we have

$$
\alpha(1 / s)=\int_{0}^{\infty} e^{-s t} g\left(e^{-s t}\right) d \alpha(t) \leq \int_{0}^{\infty} e^{-s t} 3 d \alpha(t)=3 f(s) \leq \frac{3 C}{s} \quad(0<s \leq d) .
$$

Setting $t=1 / s$ implies that

$$
\alpha(t) \leq 3 C t \quad\left(t \geq \frac{1}{d}\right) .
$$

Later on, we will let $\alpha(t)$ be the spectral counting function for the Laplacian $\Delta$.

1.3. Trivial counting lemma. Let $\Gamma$ be a cofinite Fuchsian group. Let $\pi(x)$ denote the number of primitive conjugacy classes of hyperbolic elements (of $\Gamma$ ) of norm not exceeding $x$ (the details are given below). In this section (following [Fis87, pp. 47-49]) we give an explicit constant $B$ so that for all $x \geq 1, \pi(x) \leq B x$. This result is called the trivial counting lemma 5

Let $\rho(z, w)$ denote the hyperbolic distance on $\mathbb{H}$. For $T$ hyperbolic, define the norm of $T, N(T)$, by

$$
N(T)=\exp \left(\inf _{z \in \mathbb{H}} \rho(z, T z)\right) .
$$

Note that the norm is constant within a fixed conjugacy class $\{T\}_{\Gamma}$. For $x \geq 1$ define $\pi(x)$ by

$$
\pi(x) \equiv\left|\left\{\{T\}_{\Gamma} \in \Gamma \mid N(T) \leq x\right\}\right| .
$$

Let $\mathcal{F}=\mathcal{F}_{Y} \cap \mathcal{F}_{1}^{Y} \cap \mathcal{F}_{2}^{Y} \cap \cdots \cap \mathcal{F}_{\tau}^{Y}$ be a fundamental domain for $\Gamma$, where each $\mathcal{F}_{j}^{Y}$ is a cusp sector and $\mathcal{F}_{Y}$ is compact. Let $\zeta_{1}, \ldots, \zeta_{\tau}$ be a complete list of representatives of the $\Gamma$ equivalence classes of cusps, and let $A_{1}, \ldots, A_{\tau}$ be elements of $\operatorname{PSL}(2, \mathbb{R})$ satisfying $A_{j} \zeta_{j}=\infty$. In addition, assume that $A_{j} \mathcal{F}_{j}^{Y}=[0,1) \times[Y, \infty)$.

If $\tau=1$, then it follows that $A_{1} \mathcal{F}$ has a positive Euclidean distance $\epsilon$ to the real axis $(\epsilon \leq Y)$. If $\tau \geq 2$, set

$$
\left(\begin{array}{ll}
a & b \\
c & d
\end{array}\right)=A_{2} A_{1}^{-1}
$$

Note that $c \neq 0$ since $A_{2} A_{1}^{-1}$ does not fix $\infty$; in this case set $\epsilon=\min \left\{Y, c^{-2} Y^{-1}\right\}$.

Let $K_{j} \equiv\left\{z \in A_{j} \mathcal{F} \mid \epsilon \leq \operatorname{Im}(z) \leq Y+1\right\}$. It follows that $K_{j}$ has compact closure, and hence has finite area $\left|K_{j}\right|$. Finally, let $d_{j}$ be the hyperbolic diameter of $K_{j}$.

Lemma 1.2 ([Fis87, pp. 47-49]). Let $\Gamma$ be a cofinite, non-compact Fuchsian group. Then for all $x \geq 1$,

$$
\pi(x) \leq \sum_{j=1}^{\min (2, \tau)} \frac{4 \pi}{\left|K_{j}\right|}\left(\cosh \left(\log x+2 d_{j}\right)-1\right) .
$$

Note that $\pi(x)=0$ for $x \leq 1$.

Lemma 1.3. Let

$$
B=4 \pi Y(Y+1) \sum_{j=1}^{\min (2, \tau)} e^{2 d_{j}} .
$$

\footnotetext{
${ }^{5}$ Note that the hyperbolic (or geodesic) prime number theorem implies that $\pi(x)=o(x)$ as $x \rightarrow \infty$. Hence this lemma is called trivial, the non-trivial being the prime geodesic theorem. As our goal is an absolute constant, the trivial lemma will be used in a bootstrapping argument.
} 
Then

$$
\pi(x) \leq B x \quad(x \geq 0) .
$$

Proof. Since $\pi(x)=0$ for $x \leq 1$, we assume that $x>1$. By Lemma 1.2 we must estimate

$$
\sum_{j=1}^{\min (2, \tau)} \frac{4 \pi}{\left|K_{j}\right|}\left(\cosh \left(\log x+2 d_{j}\right)-1\right) .
$$

Note that for each $j,[0,1) \times[Y, Y+1] \subset K_{j}$. Hence

$$
\left|K_{j}\right| \geq \frac{1}{Y(Y+1)} .
$$

The lemma now follows by observing that for $y, d>0$,

$$
\cosh (y+2 d)-1 \leq \cosh (y+2 d) \leq \frac{1}{2}\left(e^{y+2 d}+e^{y+2 d}\right)=e^{y+2 d},
$$

and by setting $y=\log x$.

1.4. Selberg trace formula. The main tool for this paper is the Selberg trace formula (see Hej76, Hej83, Iwa02, Ven82]).

Theorem 1.4 (Selberg trace formula).

$$
\begin{aligned}
& \sum_{n=0}^{\infty} h\left(r_{n}\right)+\frac{1}{4 \pi} \int_{\mathbb{R}} h(r) \frac{-\phi^{\prime}}{\phi}\left(\frac{1}{2}+i r\right) d r=\frac{|\mathcal{F}|}{4 \pi} \int_{\mathbb{R}} h(r) r \tanh (\pi r) d r \\
& \quad+\sum_{\{\gamma\}_{\Gamma}} \frac{\log N\left(\gamma_{0}\right)}{N(\gamma)^{1 / 2}-N(\gamma)^{-1 / 2}} g(\log N(\gamma))+\sum_{\{R\}_{\Gamma}} \frac{1}{2 m_{R} \sin \theta_{R}} \int_{\mathbb{R}} \frac{e^{-2 r \theta_{R}}}{1+e^{-2 \pi r}} h(r) d r \\
& \quad+\frac{h(0)}{4} \operatorname{tr}\left(I-\mathfrak{S}\left(\frac{1}{2}\right)\right)-\tau g(0) \log 2-\frac{\tau}{2 \pi} \int_{\mathbb{R}} h(r) \psi(1+i r) d r .
\end{aligned}
$$

Here $\lambda_{n}=\frac{1}{4}+r_{n}^{2}$ are the eigenvalues of the hyperbolic Laplacian $\Delta$ and

$$
r_{n}=\left\{\begin{array}{cc}
i \sqrt[+]{\frac{1}{4}-\lambda_{n}} & \text { if } 0 \leq \lambda_{n} \leq \frac{1}{4}, \\
\sqrt[+]{\lambda_{n}-\frac{1}{4}} & \text { if } \lambda_{n} \geq \frac{1}{4}
\end{array}\right.
$$

(see $₫ 2$ for some background material on $\Delta$ ); $h(z)$ is an even holomorphic function in the strip $|\operatorname{Im} z| \leq \frac{1}{2}+\epsilon$ satisfying

$$
|h(z)|=O\left((|z|+1)^{-2-\epsilon}\right) ;
$$

$\{\gamma\}_{\Gamma}$ are the conjugacy classes for the hyperbolic elements of $\Gamma$ with primitive generator $\gamma_{0} ; \phi(s)$ is the determinant of the scattering matrix $\mathfrak{S}(s) ;|\mathcal{F}|$ is the hyperbolic area of the fundamental domain $\mathcal{F}$ of $\Gamma ; g(r)$ is the Fourier transform of $h(z) ;\{R\}_{\Gamma}$ are conjugacy classes for the elliptic elements of $\Gamma$ with $m_{R}=|C(R)|$ (the order of the centralizer of $R$ in $\Gamma$ ) and $\operatorname{tr}(R)=2 \cos \left(\theta_{R}\right), 0<\theta_{R}<\pi$; $\tau$ is the number of cusps of $\Gamma$; and $\psi(s)$ is the logarithmic derivative of the Gamma function. See see Hej76, Hej83, Iwa02, Ven82, for more details. 


\section{HeAt Kernel For the hyperbolic Laplacian}

Let $\Gamma$ be a cofinite Fuchsian group, and let $\mathcal{H}(\Gamma)$ denote the Hilbert space of measurable functions $f: \mathbb{H} \mapsto \mathbb{C}$ satisfying

- $f(\gamma z)=f(z)$ for all $z \in \mathbb{H}, \gamma \in \Gamma$,

- $\int_{\mathcal{F}}|f(z)| d v(z)<\infty$.

Let $\Delta: \mathcal{H}(\Gamma) \mapsto \mathcal{H}(\Gamma)$ be the self-adjoint extension of the (two-dimensional hyperbolic) Laplacian. In this section we will estimate (for $0<t \leq 5$ ) the function

$$
\sum_{n=0}^{\infty} e^{-t r_{n}^{2}}+\frac{1}{4 \pi} \int_{\mathbb{R}} e^{-t r^{2}-\phi^{\prime}} \frac{1}{\phi}\left(\frac{1}{2}+i r\right) d r .
$$

For $t>0$, let

$$
\begin{aligned}
& h(r)=e^{-t r^{2}}, \\
& g(x)=\frac{1}{\sqrt{4 \pi t}} e^{-x^{2} /(4 t)} .
\end{aligned}
$$

Plugging in the pair $h, g$ into the Selberg trace formula gives an explicit formula for the (regularized) trace of the heat kernel $e^{-\Delta t}$ :

$$
\begin{aligned}
& \sum_{n=0}^{\infty} e^{-t r_{n}^{2}}+\frac{1}{4 \pi} \int_{\mathbb{R}} e^{-t r^{2}} \frac{-\phi^{\prime}}{\phi}\left(\frac{1}{2}+i r\right) d r=\frac{|\mathcal{F}|}{4 \pi} \int_{\mathbb{R}} e^{-t r^{2}} r \tanh (\pi r) d r \\
& \quad+\frac{1}{\sqrt{4 \pi t}} \sum_{\{P\}_{\Gamma}} \frac{\log N\left(P_{0}\right)}{N(P)^{1 / 2}-N(P)^{-1 / 2}} e^{-(\log N(P))^{2} /(4 t)} \\
& \quad+\sum_{\{R\}_{\Gamma}} \frac{1}{2 m_{R} \sin \theta_{R}} \int_{\mathbb{R}} \frac{e^{-2 r \theta_{R}}}{1+e^{-2 \pi r}} e^{-t r^{2}} d r+\frac{1}{4} \operatorname{tr}\left(I-\mathfrak{S}\left(\frac{1}{2}\right)\right) \\
& -\frac{\tau}{\sqrt{4 \pi t}} \log 2-\frac{\tau}{2 \pi} \int_{\mathbb{R}} e^{-t r^{2}} \psi(1+i r) d r
\end{aligned}
$$

where $\lambda_{n}=\frac{1}{4}+r_{n}^{2}$ are the eigenvalues of $\Delta$.

We will now estimate each of the terms on the right-hand side of equation (2.1) for $0<t \leq 5$.

\section{Parabolic term.}

Lemma 2.1. Let $C_{3}=8 \tau$. Then

$$
-\frac{\tau \log 2}{\sqrt{4 \pi t}}-\frac{\tau}{2 \pi} \int_{\mathbb{R}} e^{-t r^{2}} \psi(1+i r) d r+\frac{1}{4} \operatorname{tr}\left(I-\mathfrak{S}\left(\frac{1}{2}\right)\right)=\mathcal{O}_{3}\left(\frac{1}{t}\right) \quad(0<t \leq 5) .
$$

Proof. We have GR65, p. 943, 8.361]

$$
\psi(z)=\frac{\Gamma^{\prime}}{\Gamma}(z)=\log z+\int_{0}^{\infty} e^{-u z}\left(\frac{1}{u}-\frac{1}{1-e^{-u}}\right) d u \quad(\operatorname{Re}(z)>0) .
$$

Thus it follows that for $r \in \mathbb{R}$,

$$
\begin{aligned}
|\psi(1+i r)| \leq|\log (1+i r)|+\int_{0}^{\infty}\left|e^{-u(1+i r)}\left(\frac{1}{u}-\frac{1}{1-e^{-u}}\right)\right| d u \\
=|\log (1+i r)|-\int_{0}^{\infty} e^{-u}\left(\frac{1}{u}-\frac{1}{1-e^{-u}}\right) d u \\
=|\log (1+i r)|-(\psi(1)-\log 1)=|\log (1+i r)|-\gamma
\end{aligned}
$$


where $\gamma=-0.577 \ldots$ is Euler's constant. Hence

$$
|\psi(1+i r)-\log (1+i r)| \leq-\gamma .
$$

Now an elementary estimate shows that

$$
|\psi(1+i r)| \leq 4|r|^{1 / 4}+4 \quad(r \in \mathbb{R}),
$$

and it follows that

$$
\int_{\mathbb{R}} e^{-t r^{2}}\left(4|r|^{1 / 4}+4\right) d r \leq \frac{27}{t} \quad(0<t \leq 5) .
$$

Noting that

$$
\frac{\log 2}{\sqrt{4 \pi t}} \leq \frac{1 / 2}{t} \quad(0<t \leq 5)
$$

and that $\mathfrak{S}(1 / 2)$ is a unitary $(\tau \times \tau)$ matrix with real entries (hence the diagonal entries are \pm 1$)$, concludes the proof.

Elliptic term. Next, we estimate the elliptic term

$$
\sum_{\{R\}} \frac{1}{2 m_{R} \sin \theta_{R}} \int_{\mathbb{R}} \frac{e^{-2 r \theta_{R}}}{1+e^{-2 \pi r}} e^{-t r^{2}} d r,
$$

where $m_{R}$ are integers and $0<\theta_{R}<\pi$.

\section{Lemma 2.2.}

$$
\sum_{\{R\}} \frac{1}{2 m_{R} \sin \theta_{R}} \int_{\mathbb{R}} \frac{e^{-2 r \theta_{R}}}{1+e^{-2 \pi r}} e^{-t r^{2}} d r \leq \sum_{\{R\}} \frac{1}{2 m_{R} \sin \theta_{R}}\left(\frac{1}{2 \theta_{R}}+\frac{1}{2\left(\pi-\theta_{R}\right)}\right) .
$$

In particular, if

$$
C_{4}=5 \sum_{\{R\}} \frac{1}{2 m_{R} \sin \theta_{R}}\left(\frac{1}{2 \theta_{R}}+\frac{1}{2\left(\pi-\theta_{R}\right)}\right),
$$

then

$$
\sum_{\{R\}} \frac{1}{2 m_{R} \sin \theta_{R}} \int_{\mathbb{R}} \frac{e^{-2 r \theta_{R}}}{1+e^{-2 \pi r}} e^{-t r^{2}} d r=\mathcal{O}_{4}\left(\frac{1}{t}\right) \quad(0<t \leq 5) .
$$

Proof. For $0<\theta<\pi$, and $t>0$,

$$
\frac{e^{-2 r \theta}}{1+e^{-2 \pi r}} e^{-t r^{2}} \leq f_{\theta}(r) \equiv\left\{\begin{array}{cc}
e^{-2 r \theta} & r \geq 0 \\
e^{2 r(\pi-\theta)} & r<0
\end{array}\right.
$$

The lemma now follows from the equation

$$
\int_{\mathbb{R}} f_{\theta_{R}}(r) d r=\frac{1}{2 \theta_{R}}+\frac{1}{2\left(\pi-\theta_{R}\right)} .
$$




\section{Identity term.}

Lemma 2.3. For all $t>0$,

$$
\left|\frac{|\mathcal{F}|}{4 \pi} \int_{\mathbb{R}} r e^{-r^{2} t} \tanh (\pi r) d r\right| \leq \frac{|\mathcal{F}|}{4 \pi} \frac{1}{t} .
$$

Proof. Since $|\tanh (\pi r)| \leq 1$ for $r \in \mathbb{R}$,

$$
\begin{aligned}
& \frac{|\mathcal{F}|}{4 \pi} \int_{\mathbb{R}} r e^{-r^{2} t} \tanh (\pi r) d r \\
& \quad=2 \frac{|\mathcal{F}|}{4 \pi} \int_{0}^{\infty} r e^{-r^{2} t} \tanh (\pi r) d r \leq 2 \frac{|\mathcal{F}|}{4 \pi} \int_{0}^{\infty} r e^{-r^{2} t} d r=\frac{|\mathcal{F}|}{4 \pi} \frac{1}{t}
\end{aligned}
$$

Hyperbolic term. Since $\Gamma$ is discrete, there exists a hyperbolic conjugacy class $\left\{P_{s}\right\}$ with minimal norm $N\left(P_{s}\right)>1$. Choose $c>0$ so that

$$
1<c<N\left(P_{s}\right)
$$

Lemma 2.4. For $0<t \leq 5$,

$$
\frac{1}{\sqrt{4 \pi t}} \sum_{\{P\}_{\Gamma}} \frac{\log N\left(P_{0}\right)}{N(P)^{1 / 2}-N(P)^{-1 / 2}} e^{-(\log N(P))^{2} /(4 t)} \leq \frac{745 B}{t}
$$

Proof. First note that for a hyperbolic element $P$,

$$
N(P)=N\left(P_{0}\right)^{j}
$$

for some integer $j \geq 0$. Since $N(P)>1, N\left(P_{0}\right) \leq N(P)$. Noting that

$$
\frac{\log x}{x^{1 / 2}-x^{-1 / 2}} \leq 1 \quad(x>1)
$$

implies

$$
\begin{aligned}
& \frac{1}{\sqrt{4 \pi t}} \sum_{\{P\}_{\Gamma}} \frac{\log N\left(P_{0}\right)}{N(P)^{1 / 2}-N(P)^{-1 / 2}} e^{-(\log N(P))^{2} /(4 t)} \\
& \quad \leq \frac{1}{\sqrt{4 \pi t}} \sum_{\{P\}_{\Gamma}} \frac{\log N(P)}{N(P)^{1 / 2}-N(P)^{-1 / 2}} e^{-(\log N(P))^{2} /(4 t)} \\
& \quad \leq \frac{1}{\sqrt{4 \pi t}} \sum_{\{P\}_{\Gamma}} e^{-(\log N(P))^{2} /(4 t)}
\end{aligned}
$$

Rewriting the last sum as a Stieltjes integral we get

$$
\frac{1}{\sqrt{4 \pi t}} \sum_{\{P\}_{\Gamma}} e^{-(\log N(P))^{2} /(4 t)}=\frac{1}{\sqrt{4 \pi t}} \int_{c}^{\infty} e^{-(\log x)^{2} /(4 t)} d \pi(x) .
$$


Integrating by parts, and the trivial counting lemma (Lemma 1.3) yield

$$
\begin{aligned}
\frac{1}{\sqrt{4 \pi t}} \int_{c}^{\infty} e^{-(\log x)^{2} /(4 t)} d \pi(x) & \leq \frac{1}{\sqrt{4 \pi t}} \int_{c}^{\infty} \frac{\log x}{2 t x} e^{-(\log x)^{2} /(4 t)} B x d x \\
& =\frac{B}{2 t} \frac{1}{\sqrt{4 \pi t}} \int_{c}^{\infty}(\log x) e^{-(\log x)^{2} /(4 t)} d x \\
& \leq \frac{B}{2 t} \frac{1}{\sqrt{4 \pi t}} \int_{1}^{\infty}(\log x) e^{-(\log x)^{2} /(4 t)} d x \\
& =B\left(\frac{1}{2} e^{t} \operatorname{erf}(\sqrt{t})+\frac{1}{2} e^{t}+\frac{1}{\sqrt{4 \pi t}}\right) \\
& \leq B\left(e^{t}+\frac{1}{\sqrt{4 \pi t}}\right) \\
& \leq \frac{745 B}{t}(0<t \leq 5) .
\end{aligned}
$$

The last estimate is a trivial estimate.

Spectral terms. We have now bounded all the terms on the right side of the trace formula. By Lemmas 2.1, 2.2, 2.3, and 2.4, we have

Lemma 2.5. Let

$$
C_{5}=8 \tau+C_{4}+\frac{|\mathcal{F}|}{4 \pi}+745 B
$$

Then

$$
\left|\sum_{n=0}^{\infty} e^{-t r_{n}^{2}}+\frac{1}{4 \pi} \int_{\mathbb{R}} e^{-t r^{2}} \frac{-\phi^{\prime}}{\phi}\left(\frac{1}{2}+i r\right) d r\right|=\mathcal{O}_{5}\left(\frac{1}{t}\right) \quad(0<t \leq 5) .
$$

\section{Explicit bound For the SPECtRal COUNTing FUnCtion}

We would like to apply Lemma 1.1 to the equation

$$
\left|\sum_{n=0}^{\infty} e^{-t r_{n}^{2}}+\frac{1}{4 \pi} \int_{\mathbb{R}} e^{-t r^{2}} \frac{-\phi^{\prime}}{\phi}\left(\frac{1}{2}+i r\right) d r\right|=\mathcal{O}_{5}\left(\frac{1}{t}\right) \quad(0<t \leq 5)
$$

but a minor problem stops us: The real-valued function $\frac{-\phi^{\prime}}{\phi}\left(\frac{1}{2}+i r\right)$ may be negative for some values of $r$. However, at the cost of a small error term, we will (following Selberg) replace the signed function $\frac{-\phi^{\prime}}{\phi}\left(\frac{1}{2}+i r\right)$ with a non-negative function.

Notation. Recall from $\$ 1.3$ that $\zeta_{1}, \ldots, \zeta_{\tau}$ are a complete list of representatives of the $\Gamma$ equivalence classes of cusps, and $A_{1}, \ldots, A_{\tau}$ are elements of $\operatorname{PSL}(2, \mathbb{R})$ satisfying $A_{j} \zeta_{j}=\infty$. Set

$$
\Gamma^{i} \equiv A_{i} \Gamma A_{i}^{-1} .
$$

It follows that $\infty$ is a cusp for $\Gamma^{i}$.

For each $\gamma \in \operatorname{PSL}(2, \mathbb{R})$ let $c(\gamma)$ denote the lower left-hand matrix entry of $\gamma$. Now set

$$
\Delta_{i} \equiv\left\{\gamma \in \Gamma^{i} \mid \gamma(\infty) \neq \infty\right\}
$$

and let

$$
c_{1}=\min _{i=1 \ldots \tau}\left\{|c(\gamma)| \mid \gamma \in \Delta_{i}\right\} .
$$


Since $\Gamma$ is discrete, Shimizu's Lemma EGM98, p. 48] assert 6 that $c_{1}>0$.

Modified scattering matrix. Recall from $\$ 1.4$ that $\phi(s)$ is the determinant of the scattering matrix. Let $\sigma_{1} \geq \sigma_{2} \geq \cdots \geq \sigma_{\mathcal{N}}$ be all the poles of $\phi(s)$ in the interval $\left(\frac{1}{2}, 1\right]$, and let

$$
\phi_{r}(s) \equiv c_{1}^{2 s-1} \phi(s) \prod_{j=1}^{\mathcal{N}} \frac{s-\sigma_{j}}{s-1+\sigma_{j}}
$$

Lemma 3.1. For all $r \in \mathbb{R}$,

(1) $\frac{-\phi_{r}^{\prime}}{\phi_{r}}\left(\frac{1}{2}+i r\right)$ is non-negative.

$$
\left|\frac{-\phi^{\prime}}{\phi}\left(\frac{1}{2}+i r\right)-\frac{-\phi_{r}^{\prime}}{\phi_{r}}\left(\frac{1}{2}+i r\right)\right| \leq 2\left|\log c_{1}\right|+\frac{\mathcal{N}}{\left(\sigma_{\mathcal{N}}-\frac{1}{2}\right)^{2}+r^{2}} .
$$

Proof. Item (1) is proved in Ven82, Theorem 3.5.5]. (2) follows from elementary estimates: Taking the logarithmic derivative of both sides of the equation

$$
\theta_{r}(s)=c_{1}^{2 s-1} \phi(s) \prod_{j=1}^{\mathcal{N}} \frac{s-\sigma_{j}}{s-1+\sigma_{j}}
$$

plugging in $s=\frac{1}{2}+i r$, and recalling that $\frac{1}{2}<\sigma_{\mathcal{N}} \leq \cdots \leq \sigma_{1} \leq 1$, we obtain

$$
\begin{aligned}
& \left|\frac{-\phi^{\prime}}{\phi}\left(\frac{1}{2}+i r\right)-\frac{-\phi_{r}^{\prime}}{\phi_{r}}\left(\frac{1}{2}+i r\right)\right|=\left|-2 \log c_{1}+\sum_{j=1}^{\mathcal{N}} \frac{1}{\left(-\frac{1}{2}+i r-\sigma_{j}\right)\left(\frac{1}{2}+i r-\sigma_{j}\right)}\right| \\
& \quad \leq\left|2 \log c_{1}\right|+\sum_{j=1}^{\mathcal{N}} \frac{1}{\left(\sigma_{\mathcal{N}}-\frac{1}{2}\right)^{2}+r^{2}}=2\left|\log c_{1}\right|+\frac{\mathcal{N}}{\left(\sigma_{\mathcal{N}}-\frac{1}{2}\right)^{2}+r^{2}} .
\end{aligned}
$$

Let

$$
\begin{gathered}
W(\lambda)=\left\{\begin{array}{cc}
\frac{1}{4 \pi} \int_{-\sqrt{\lambda-\frac{1}{4}}}^{\sqrt{\lambda-\frac{1}{4}}}\left|\frac{\phi^{\prime}}{\phi}\left(\frac{1}{2}+i r\right)\right| d r, & \lambda \geq \frac{1}{4}, \\
0, & 0 \leq \lambda \leq \frac{1}{4},
\end{array}\right. \\
Q(\lambda)=\left\{\begin{array}{cc}
\frac{1}{4 \pi} \int_{-\sqrt{\lambda-\frac{1}{4}}}^{\sqrt{\lambda-\frac{1}{4}}} \frac{-\phi_{r}^{\prime}}{\phi_{r}}\left(\frac{1}{2}+i r\right) d r, & \lambda \geq \frac{1}{4}, \\
0, & 0 \leq \lambda \leq \frac{1}{4},
\end{array}\right.
\end{gathered}
$$

and let $N(\lambda)=\left|\left\{\lambda_{n} \mid \lambda_{n} \leq \lambda\right\}\right|$.

Lemma 3.2. Let

$$
\begin{aligned}
& C_{6}=C_{5}+\left(2\left|\log c_{1}\right|+\frac{\mathcal{N}}{\left(\sigma_{\mathcal{N}}-\frac{1}{2}\right)^{2}}\right) \frac{\sqrt{5}}{\sqrt{16 \pi}} \\
& C_{7}=\frac{8}{4 \pi}\left(2\left|\log c_{1}\right|+\frac{\mathcal{N}}{\left(\sigma_{\mathcal{N}}-\frac{1}{2}\right)^{2}}\right) .
\end{aligned}
$$

Then the following statements hold:

(1) $\int_{0}^{\infty} e^{-t \lambda} d(N(\lambda)+Q(\lambda)) \leq \frac{C_{6}}{t} \quad(0<t \leq 5)$.

(2) $N(\lambda)+Q(\lambda) \leq 3 C_{6} \lambda \quad\left(\lambda \geq \frac{1}{4}\right)$.

${ }^{6}$ For $\operatorname{PSL}(2, \mathbb{Z}), c_{1}=1$. 
(3) $N(\lambda)+W(\lambda) \leq\left(3 C_{6}+C_{7}\right) \lambda \quad\left(\lambda \geq \frac{1}{4}\right)$.

Proof. (1) A simple calculation using $\lambda_{n}=\frac{1}{4}+r_{n}^{2}$ shows that

$$
\int_{0}^{\infty} e^{-t \lambda} d(N(\lambda)+Q(\lambda))=e^{-t / 4}\left(\sum_{n=0}^{\infty} e^{-t r_{n}^{2}}+\frac{1}{4 \pi} \int_{\mathbb{R}} e^{-t r^{2}} \frac{-\phi_{r}^{\prime}}{\phi_{r}}\left(\frac{1}{2}+i r\right) d r\right) .
$$

Since $e^{-t / 4} \leq 1$

$$
\int_{0}^{\infty} e^{-t \lambda} d(N(\lambda)+Q(\lambda)) \leq\left(\sum_{n=0}^{\infty} e^{-t r_{n}^{2}}+\frac{1}{4 \pi} \int_{\mathbb{R}} e^{-t r^{2}} \frac{-\phi_{r}^{\prime}}{\phi_{r}}\left(\frac{1}{2}+i r\right) d r\right) .
$$

Note that both sides of the above inequality are positive. Let

$$
U(r)=\frac{-\phi^{\prime}}{\phi}\left(\frac{1}{2}+i r\right)-\frac{-\phi_{r}^{\prime}}{\phi_{r}}\left(\frac{1}{2}+i r\right) .
$$

Then rewriting (3.1) gives us

$$
\left|\sum_{n=0}^{\infty} e^{-t r_{n}^{2}}+\frac{1}{4 \pi} \int_{\mathbb{R}} e^{-t r^{2}}\left(\frac{-\phi_{r}^{\prime}}{\phi_{r}}\left(\frac{1}{2}+i r\right)+U(r)\right) d r\right| \leq \frac{C_{5}}{t} \quad(0<t \leq 5) .
$$

But Lemma 3.1] and a crude estimate (see equation (3.6) for the idea of the estimate), show that

$$
\frac{1}{4 \pi} \int_{\mathbb{R}} e^{-t r^{2}}|U(r)| d r \leq\left(2\left|\log c_{1}\right|+\frac{\mathcal{N}}{\left(\sigma_{\mathcal{N}}-\frac{1}{2}\right)^{2}}\right) \frac{1}{\sqrt{16 \pi}} \frac{1}{\sqrt{t}},
$$

and since

$$
\frac{1}{\sqrt{t}} \leq \frac{\sqrt{5}}{t} \quad(0<t \leq 5)
$$

it follows that

$$
\begin{aligned}
& \left|\sum_{n=0}^{\infty} e^{-t r_{n}^{2}}+\frac{1}{4 \pi} \int_{\mathbb{R}} e^{-t r^{2}} \frac{-\phi_{r}^{\prime}}{\phi_{r}}\left(\frac{1}{2}+i r\right) d r\right| \\
& \quad \leq \frac{C_{5}+\left(2\left|\log c_{1}\right|+\frac{\mathcal{N}}{\left(\sigma_{\mathcal{N}}-\frac{1}{2}\right)^{2}}\right) \frac{\sqrt{5}}{\sqrt{16 \pi}}}{t} \quad(0<t \leq 5) .
\end{aligned}
$$

(1) is now proved.

(2) follows from the fact that $N(\lambda)+Q(\lambda)$ is non-decreasing, (1), and Lemma 1.1

To prove (3), note that (3.3) implies that

$$
N(\lambda)+W(\lambda) \leq N(\lambda)+Q(\lambda)+\frac{1}{4 \pi} \int_{-\sqrt{\lambda-\frac{1}{4}}}^{\sqrt{\lambda-\frac{1}{4}}}|U(t)| d t .
$$

Next, using Lemma 3.1, a trivial estimate gives

$$
\begin{aligned}
\int_{-\sqrt{\lambda-\frac{1}{4}}}^{\sqrt{\lambda-\frac{1}{4}}}|U(t)| d t \leq \int_{-\sqrt{\lambda-\frac{1}{4}}}^{\sqrt{\lambda-\frac{1}{4}}}\left(2\left|\log c_{1}\right|+\frac{\mathcal{N}}{\left(\sigma_{\mathcal{N}}-\frac{1}{2}\right)^{2}+r^{2}}\right) d t \\
\leq \int_{-\sqrt{\lambda-\frac{1}{4}}}^{\sqrt{\lambda-\frac{1}{4}}}\left(2\left|\log c_{1}\right|+\frac{\mathcal{N}}{\left(\sigma_{\mathcal{N}}-\frac{1}{2}\right)^{2}}\right) d t
\end{aligned}
$$


(3) now follows from the equation

$$
\sqrt{\lambda+\frac{1}{4}} \leq 4 \lambda \quad(\lambda \geq 1 / 4)
$$

3.1. Implied constant for the spectral counting function. Recall that $\lambda_{n}=$ $\frac{1}{4}+r_{n}^{2}$. Also note that for $\lambda_{n} \geq \frac{1}{4}$, we have $r_{n} \geq 0$.

Let

$$
\mu(r)=\left|\left\{r_{n} \mid 0 \leq r_{n} \leq r\right\}\right|+\frac{1}{4 \pi} \int_{-r}^{r}\left|\frac{\phi^{\prime}}{\phi}\left(\frac{1}{2}+i t\right)\right| d t .
$$

Lemma 3.2(3) immediately implies that

$$
\mu(r) \leq N\left(r^{2}+\frac{1}{4}\right)+W\left(r^{2}+\frac{1}{4}\right) \leq\left(3 C_{6}+C_{7}\right)\left(r^{2}+\frac{1}{4}\right) \quad(r \geq 0) .
$$

We summarize our result:

Theorem 3.3. Let $C=3 C_{6}+C_{7}$. Then

$$
\mu(r) \leq C\left(r^{2}+\frac{1}{4}\right) \quad(r \geq 0) .
$$

3.2. Explicit estimate for the modular group. Let $\Gamma=\operatorname{PSL}(2, \mathbb{Z})$. In this section we will give an explicit upper bound for the number $C$ which depends on $B, C_{3}, C_{4}, C_{5}, C_{6}$, and $C_{7}$.

The constant $B$. From the trivial counting lemma ( $(1.3)$ we have

$$
B=4 \pi Y(Y+1) \sum_{j=1}^{\min (2, \tau)} e^{2 d_{j}},
$$

where $\tau=1$. Let $\mathcal{F}$ be the standard fundamental domain of $\Gamma$ such that

$$
\mathcal{F}=\left\{z \in \mathbb{H}\left|-\frac{1}{2} \leq \operatorname{Re} z \leq \frac{1}{2},\right| z \mid \geq 1\right\} .
$$

The euclidian distance of $\mathcal{F}$, to the $x$-axis, is $\epsilon=\frac{\sqrt{3}}{2}$. We can decompose $\mathcal{F}=$ $\mathcal{F}_{0} \cup \mathcal{F}(Y)$, where

$$
\mathcal{F}(Y)=\left\{z \in \mathbb{H} \mid-\frac{1}{2} \leq \operatorname{Re} z \leq \frac{1}{2}, \operatorname{Im} z>Y\right\},
$$

with $Y=2$.

Recall that $K_{1}=\left\{z \in \mathcal{F} \mid \frac{3}{2} \leq \operatorname{Im}(z) \leq Y+1\right\}$. Let $d_{1}$ be the hyperbolic diameter of $K_{1}$. To estimate $d_{1}$ note that $\cosh (\rho(z, w))=1+\frac{|z-w|^{2}}{\operatorname{Im} z \operatorname{Im} w}$, where $\rho(z, w)$ is the hyperbolic distance between $z$ and $w$. An elementary calculation shows that $d_{1}<2.3$. Hence $B<753$.

The constant $C_{4}$. It is known that $\Gamma$ has two classes of elliptic elements, represented by

$$
R=\left(\begin{array}{cc}
0 & -1 \\
1 & 0
\end{array}\right)
$$

and

$$
S=\left(\begin{array}{cc}
1 & -1 \\
1 & 0
\end{array}\right)
$$

Elementary calculations show that $m_{R}=2$ (the order of the centralizer of $R$ in $\Gamma$ ) and that $0=2 \cos \left(\theta_{R}\right)$ where $0<\theta_{R}<\pi$; thus $\theta_{R}=\frac{\pi}{2}$. Similarly, $m_{S}=3$ and $\theta_{S}=\frac{\pi}{3}$. From Lemma 2.2 it follows that $C_{4}<\frac{3}{2}$. 
The remaining constants. Now $C_{3}=8 \tau=8$, and $|\mathcal{F}|=\frac{\pi}{3}$.

The number $c_{1}=1$ and there is only one pole (at $s=1$ ) for the scattering matrix $\phi(s)$, so $\mathcal{N}=1$ and $\sigma_{\mathcal{N}}=1$. Now $C_{6}, C_{7}$, and $C$ can be easily computed. The result is

Theorem 3.4. Let $\Gamma=\operatorname{PSL}(2, \mathbb{Z})$. Let $\mu(r)$ be the spectral counting function (3.7), and let

$$
C=1,682,997 \text {. }
$$

Then for $r \geq 0$,

$$
\mu(r) \leq C\left(r^{2}+\frac{1}{4}\right) .
$$

The estimate for $C$ is probably not sharp. However, we did not use any of the number theoretic properties of $\operatorname{PSL}(2, \mathbb{Z})$ to arrive at the estimate; we only assumed that $\Gamma$ was a cofinite Fuchsian group.

\section{Bound For the Huber constant}

In this section, we adapt Randol's proof (Ran84) of the prime geodesic theorem to an arbitrary cofinite Fuchsian group. Along the way, using Theorem 3.3, we will obtain an upper bound for the Huber constant $C_{M}$. Our main references are Ran84, pp. 295-300] and Bus92, Section 9.6].

4.1. Explicit bump function. For $T>0$ set

$$
I_{T}(x)= \begin{cases}1 & |x| \leq T \\ 0 & |x|>T\end{cases}
$$

For functions $f, g$ on $\mathbb{R}$, we set

$$
\widehat{f}(x) \equiv \frac{1}{\sqrt{2 \pi}} \int_{\mathbb{R}} f(y) e^{-i x y} d y
$$

and

$$
(f * g)(x) \equiv \frac{1}{\sqrt{2 \pi}} \int_{\mathbb{R}} f(x-y) g(y) d y .
$$

Lemma 4.1. There exists a non-negative function $\phi(x)$ with the following properties:

(1) $\phi \in C_{0}^{\infty}(\mathbb{R})$ with $\operatorname{supp}(\phi) \subset[-1,1]$.

(2) $\widehat{\phi}(0)=1$.

(3) Let $C_{1}=2(e-1)$. Then $\widehat{\phi}(\epsilon z)-1=\mathcal{O}_{1}(\epsilon)$ for $0<\epsilon \leq 1$, and $z \in i\left[-\frac{1}{2}, 1\right]$.

(4) Let $b>0, C_{2}^{(b)}=\frac{848}{\sqrt{2 \pi}} e^{b}$. Then for $z=r+i t,|t| \leq b$, we have

$$
\widehat{\phi}(z)=\mathcal{O}_{2}\left((1+|r|)^{-2}\right), \quad(r \in \mathbb{R},|t| \leq b) .
$$

Proof. Let

$$
c_{0}=\int_{-1}^{1} \exp \left(\frac{1}{x^{2}-1}\right) d x=0.4439938 \ldots,
$$

and let

$$
\phi(x)=\frac{\sqrt{2 \pi}}{c_{0}}\left\{\begin{array}{cl}
\exp \left(\frac{1}{x^{2}-1}\right) & |x|<1 \\
0 & |x| \geq 1 .
\end{array}\right.
$$

(11) and (2) now follow from the definition of $\phi$. 
For $w \in \mathbb{C},|w| \leq 1$, observe that

$$
\begin{aligned}
\left|e^{w}-1\right| & =\left|w+\frac{w^{2}}{2}+\frac{w^{3}}{3 !}+\ldots,\right|=|w| \cdot\left|1+\frac{|w|}{2}+\frac{|w|^{2}}{3 !}+\ldots,\right| \\
& \leq|w| \cdot\left|1+\frac{1}{2}+\frac{1}{3 !}+\ldots,\right|=(e-1)|w| .
\end{aligned}
$$

For $0<\epsilon \leq 1$ and $z \in i\left[-\frac{1}{2}, 1\right]$, note that $|\epsilon z| \leq 1$. Let $w=\epsilon z$. By the definition and (2), it follows that

$$
\begin{aligned}
& \widehat{\phi}(\epsilon z)-1=\frac{1}{\sqrt{2 \pi}} \int_{\mathbb{R}} \phi(y) e^{-i w y} d y-1 \\
& =\frac{1}{\sqrt{2 \pi}} \int_{\mathbb{R}} \phi(y) e^{-i w y} d y-\frac{1}{\sqrt{2 \pi}} \int_{\mathbb{R}} \phi(y) d y=\frac{1}{\sqrt{2 \pi}} \int_{-1}^{1} \phi(y)\left(e^{-i w y}-1\right) d y .
\end{aligned}
$$

Now, since

it follows that

$$
\frac{\phi(y)}{\sqrt{2 \pi}} \leq 1 \quad(y \in \mathbb{R})
$$

$$
\begin{aligned}
|\widehat{\phi}(\epsilon z)-1| & \leq \int_{-1}^{1}\left|e^{-i w y}-1\right| d y \\
& \leq \int_{-1}^{1}(e-1)|w| d y=2(e-1)|w|=2(e-1)|\epsilon z| \leq 2(e-1) \epsilon .
\end{aligned}
$$

This proves (3).

Next we prove (4). Suppose $|\operatorname{Im}(z)| \leq b$. Then since $\widehat{\phi}(0)=1$ it follows that

$$
|\widehat{\phi}(z)| \leq e^{b} .
$$

Integrating by parts, twice, shows that for $z \neq 0$,

$$
|\widehat{\phi}(z)| \leq \frac{1}{|z|^{2}} \frac{1}{\sqrt{2 \pi}} e^{b} \int_{-1}^{1}\left|\phi^{\prime \prime}(y)\right| d y .
$$

Now, a simple calculation 7 shows that

$$
\left|\phi^{\prime \prime}(y)\right| \leq 106 \quad(-1 \leq y \leq 1) .
$$

Hence, for $z \neq 0$,

Finally, observing that

$$
|\widehat{\phi}(z)| \leq \frac{1}{|z|^{2}} \frac{212}{\sqrt{2 \pi}} e^{b}
$$

and

$$
1 \leq \frac{2}{1+|z|^{2}} \quad(|z| \leq 1)
$$

it follows that

$$
\frac{1}{|z|^{2}} \leq \frac{2}{1+|z|^{2}} \quad(|z| \geq 1)
$$

$$
|\widehat{\phi}(|z|)| \leq \frac{1}{|z|^{2}+1} \frac{424}{\sqrt{2 \pi}} e^{b} \leq \frac{1}{r^{2}+1} \frac{424}{\sqrt{2 \pi}} e^{b} \leq \frac{1}{(|r|+1)^{2}} \frac{848}{\sqrt{2 \pi}} e^{b}
$$

${ }^{7}$ First note that $\phi^{\prime \prime}(x)=\frac{\phi(x)}{\left(x^{2}-1\right)^{4}}\left(3 x^{4}-1\right)$. Next, using elementary calculus maximize $\frac{\phi(x)}{\left(x^{2}-1\right)^{4}}$ and $\left(3 x^{4}-1\right)$, separately, and multiply their maximums. Of course, this argument does not yield the optimal bound. 
since

$$
\frac{1}{r^{2}+1} \leq \frac{2}{(|r|+1)^{2}} \quad(r \neq 0)
$$

This concludes the proof.

4.2. Explicit lemmas. In this section we prove some lemmas which allow us to obtain explicit constants from Randol's proof of the prime geodesic theorem. Recall (๕4.1), and the definitions of $I_{T}(x)$ and $\phi$. For $\epsilon>0$, define

$$
\phi_{\epsilon}(x) \equiv \epsilon^{-1} \phi\left(\epsilon^{-1} x\right) \text {, }
$$

and define

$$
g_{T}^{\epsilon}(x) \equiv\left(2 \cosh \left(\frac{x}{2}\right)\right)\left(I_{T} * \phi_{\epsilon}\right)(x) .
$$

Set

$$
h_{T}^{\epsilon}(r)=\widehat{g_{T}^{\epsilon}}(r) .
$$

It follows that

$$
h_{T}^{\epsilon}(r)=S\left(r+\frac{i}{2}\right)+S\left(r-\frac{i}{2}\right)
$$

where

$$
S(w)=\left(\frac{2}{w} \sin (T w)\right) \widehat{\phi}_{\epsilon}(w), \quad S(0)=2 T .
$$

Note that

$$
\widehat{\phi}_{\epsilon}(w)=\widehat{\phi}(\epsilon w) .
$$

The main idea behind the proof is to apply the Selberg trace formula to the pair $h_{T}^{\epsilon}(r), g_{T}^{\epsilon}(x)$, and to extract the leading and error term from the trace formula. Much of our effort will be spent on giving explicit estimates to each term in the trace formula.

We next set up some important notation. For $0 \leq \lambda_{k} \leq \frac{1}{4}$, a small eigenvalue, define $r_{k}$ and $s_{k}$, by

$$
r_{k} \equiv i \sqrt[+]{\frac{1}{4}-\lambda_{k}} \equiv i\left(s_{k}-\frac{1}{2}\right) .
$$

Let $\mathcal{A}$ denote the number small eigenvalues:

$$
\mathcal{A} \equiv\left|\left\{\lambda_{k} \mid \lambda_{k} \leq \frac{1}{4}\right\}\right| .
$$

Explicitly, $0=\lambda_{0}<\lambda_{1} \leq \cdots \leq \lambda_{\mathcal{A}} \leq \frac{1}{4}$.

Lemma 4.2. Let $C_{10}=10 \frac{848}{\sqrt{2 \pi}} \sqrt{e}$, then for $r \geq 0,0<\epsilon \leq 1$,

(1) $\left|h_{T}^{\epsilon}(r)\right| \leq C_{10} e^{T / 2}(1+r)^{-1}(1+\epsilon r)^{-2}$.

(2) For $r \in\left[0, \frac{1}{\epsilon}\right]$,

$$
\left|h_{T}^{\epsilon}(r)\right| \leq C_{10} \frac{e^{T / 2}}{1+r}
$$

(3) For $r \in\left[\frac{1}{\epsilon}, \infty\right)$,

$$
\left|h_{T}^{\epsilon}(r)\right| \leq C_{10} \frac{e^{T / 2}}{\epsilon^{2} r^{3}}
$$


Proof. We prove (1); (2) and (3) will then follow from elementary estimates.

Recall that for $r \geq 0$,

$$
h_{T}^{\epsilon}(r)=S\left(r+\frac{i}{2}\right)+S\left(r-\frac{i}{2}\right)
$$

where

$$
S(w)=\left(\frac{2}{w} \sin (T w)\right) \widehat{\phi}_{\epsilon}(w),
$$

and note that $\widehat{\phi}_{\epsilon}(w)=\widehat{\phi}(\epsilon w)$. By Part 4 of Lemma 4.1, with $b=1 / 2$, it follows that

where

$$
\widehat{\phi}\left(\epsilon\left(r \pm \frac{i}{2}\right)\right)=O_{2}\left((1+\epsilon r)^{-2}\right)
$$

Now

$$
C_{2} \equiv C_{2}^{(1 / 2)}=\frac{848}{\sqrt{2 \pi}} \sqrt{e} \text {. }
$$

$$
\frac{2}{\left|r \pm \frac{i}{2}\right|} \leq \frac{5}{1+r}
$$

and

$$
\left|\sin T\left(r \pm \frac{i}{2}\right)\right| \leq e^{T / 2}
$$

Hence,

$$
\left|h_{T}^{\epsilon}(r)\right| \leq 2 \frac{5}{1+r} C_{2}(1+\epsilon r)^{-2} .
$$

The next lemma will be used repeatedly.

Lemma 4.3. Let $u(r)$ be a non-decreasing function on $[0, \infty)$ that satisfies

$$
|u(r)| \leq c r^{2}+b \quad(r \geq 0) ; c, b>0 .
$$

Then

where $K=C_{10}\left(6 c+\frac{10 b}{3}\right)$.

$$
\int_{0}^{\infty}\left|h_{T}^{\epsilon}(r)\right| d u(r) \leq K \frac{e^{T / 2}}{\epsilon},
$$

Proof. By Lemma 4.2, it suffices to estimate

$$
\int_{0}^{1 / \epsilon} \frac{1}{1+r} d u(r) \text { and } \int_{1 / \epsilon}^{\infty} \frac{1}{r^{3}} d u(r) .
$$

Integrating by parts, yields

$$
\begin{aligned}
\int_{0}^{1 / \epsilon} \frac{1}{1+r} d u & (r)=\left[\frac{u(r)}{1+r}\right]_{0}^{1 / \epsilon}+\int_{0}^{1 / \epsilon} \frac{u(r)}{(1+r)^{2}} d r \\
\leq & \frac{c / \epsilon^{2}+b}{1+1 / \epsilon}+b+\int_{0}^{1 / \epsilon} \frac{c r^{2}+b}{(1+r)^{2}} d r \leq \frac{c}{\epsilon}+b+\frac{3 c}{\epsilon}+\frac{b}{\epsilon} \leq \frac{4 c+2 b}{\epsilon} .
\end{aligned}
$$

A similar argument shows that

$$
\int_{1 / \epsilon}^{\infty} \frac{1}{r^{3}} d u(r) \leq(c+b) \epsilon+\left(c+\frac{b}{3}\right) \epsilon=\left(2 c+\frac{4 b}{3}\right) \epsilon .
$$

Hence,

$$
\int_{0}^{\infty}\left|h_{T}^{\epsilon}(r)\right| d u(r) \leq C_{10}\left(6 c+\frac{10 b}{3}\right) \frac{e^{T / 2}}{\epsilon}
$$


4.3. The leading term of the prime geodesic theorem. In this section we show that the sum

$$
\sum_{\lambda_{k} \leq \frac{1}{4}} h_{T}^{\epsilon}\left(r_{k}\right)
$$

gives us the leading term of the prime geodesic theorem. In the next section, we will see that the above sum is a term in the Selberg trace formula.

Lemma 4.4. Let $\epsilon=e^{-T / 4}$, and

$$
C_{12} \equiv(\mathcal{A}-1)\left(1+3 C_{1}+\frac{2}{1-s_{1}}\left(1+C_{1}\right)\right)+2 C_{1}+2 .
$$

Then

where

$$
\left|\sum_{\lambda_{k} \leq \frac{1}{4}} h_{T}^{\epsilon}\left(r_{k}\right)-\sum_{\lambda_{k} \leq \frac{1}{4}} \frac{e^{s_{k} T}}{s_{k}}\right|=\mathcal{O}_{12}\left(e^{3 T / 4}\right),
$$

$$
s_{1} \equiv\left\{\begin{array}{cc}
\frac{1}{2}+\sqrt[+]{\frac{1}{4}-\lambda_{1}} & \lambda_{1} \leq \frac{1}{4} \\
\infty & \lambda_{1}>\frac{1}{4}
\end{array}\right.
$$

Proof. First we deal with the case $\lambda_{0}=0$. We have

$$
\begin{aligned}
h_{T}^{\epsilon}\left(r_{0}\right)=h_{T}^{\epsilon}\left(\frac{i}{2}\right) & =\frac{2}{i} \sin (i T) \widehat{\phi}(i \epsilon)+2 T=\widehat{\phi}(i \epsilon)\left(e^{T}-e^{-T}\right)+2 T \\
& =\left(1+\mathcal{O}_{1}(\epsilon)\right)\left(e^{T}-e^{-T}\right)+2 T .
\end{aligned}
$$

Hence,

$\left|h_{T}^{\epsilon}\left(r_{0}\right)-e^{T}\right| \leq C_{1} \epsilon e^{T}+e^{-T}\left|\mathcal{O}_{1}(\epsilon)\right|+2 T \leq C_{1} \epsilon e^{T}+1+C_{1} \epsilon+2 T \leq\left(2 C_{1}+2\right) e^{3 T / 4}$.

The last inequality follows from the trivial bound $1+2 T \leq 2 e^{3 T / 4}$, and by noting that $\epsilon=e^{-T / 4}$.

Next, we handle the (possible) case of non-zero small eigenvalues. Let $0<k \leq \mathcal{A}$,

$$
\lambda=\lambda_{k}, \quad r=r_{k}, \quad s=s_{k} .
$$

It follows that

$$
h_{T}^{\epsilon}(r)=\frac{\widehat{\phi}(i \epsilon s)}{s}\left(e^{s T}-e^{-s T}\right)+\frac{\widehat{\phi}(i \epsilon(s-1))}{s-1}\left(e^{(s-1) T}-e^{-(s-1) T}\right) .
$$

Here, $\frac{1}{2} \leq s \leq s_{1}<1$. By Lemma 4.1, Part 3 ,

$$
\widehat{\phi}(i \epsilon s)=1+\mathcal{O}_{1}(\epsilon) \text { and } \widehat{\phi}(i \epsilon(s-1))=1+\mathcal{O}_{1}(\epsilon) .
$$

Therefore,

$$
\begin{aligned}
\left|h_{T}^{\epsilon}(r)-\frac{e^{s T}}{s}\right| & \leq \frac{e^{s T}}{s}+\left(1+\left|\mathcal{O}_{1}(\epsilon)\right|\right) e^{-s T}+\frac{1}{|s-1|}\left(1+e^{T / 2}\right)\left(1+\left|\mathcal{O}_{1}(\epsilon)\right|\right) \\
& \leq \frac{1}{\frac{1}{2}} e^{T} C_{1} \epsilon+\left(1+C_{1} \epsilon\right)+\frac{1}{1-s_{1}}\left(1+e^{T / 2}\right)\left(1+C_{1} \epsilon\right) .
\end{aligned}
$$

Next, set $\epsilon=e^{-T / 4}$. We obtain

$$
\left|h_{T}^{\epsilon}(r)-\frac{e^{s T}}{s}\right| \leq\left(1+3 C_{1}+\frac{2}{1-s_{1}}\left(1+C_{1}\right)\right) e^{3 T / 4} .
$$


Then, finally, we have

$$
\begin{aligned}
& \left|\sum_{\lambda_{k} \leq \frac{1}{4}} h_{T}^{\epsilon}\left(r_{k}\right)-\sum_{\lambda_{k} \leq \frac{1}{4}} \frac{e^{s_{k} T}}{s_{k}}\right| \\
& \quad \leq\left[(\mathcal{A}-1)\left(1+3 C_{1}+\frac{2}{1-s_{1}}\left(1+C_{1}\right)\right)+2 C_{1}+2\right] e^{3 T / 4} .
\end{aligned}
$$

Of course, if there are no non-zero small eigenvalues, then $\mathcal{A}=1$.

4.4. Application of the trace formula. Let $\Gamma$ be a cofinite Fuchsian group, and let $M=\Gamma \backslash \mathbb{H}$ be the corresponding hyperbolic orbifold. Let $\mathcal{C}(M)$ denote the set of closed geodesics of $M$, and let $\mathcal{P}(M)$ denote the set of prime (or primitive) closed geodesics (see Bus92, p. 245]). For each $\gamma \in \mathcal{C}(M)$ there exists a unique prime geodesic $\gamma_{0}$ and a unique exponent $m \geq 1$ so that $\gamma=\gamma_{0}^{m}$. Let $l(\gamma)$ denote the length of $\gamma$. Associated to $\gamma$ is a unique hyperbolic conjugacy class $\left\{P_{\gamma}\right\}_{\Gamma}$ with norm

$$
N_{\gamma} \equiv N\left(P_{\gamma}\right)=e^{l(\gamma)}
$$

For $\gamma \in \mathcal{C}(M)$ define

$$
\Lambda(\gamma) \equiv \log N_{\gamma_{0}}=l\left(\gamma_{0}\right)
$$

Let

$$
\begin{aligned}
H_{\epsilon}(T) & =\sum_{\gamma \in \mathcal{C}(M)} \frac{\Lambda(\gamma)}{N_{\gamma}^{1 / 2}-N_{\gamma}^{-1 / 2}} g_{T}^{\epsilon}(x), \\
H(T) & =\sum_{l(\gamma) \leq T} \Lambda(\gamma) \frac{1+N_{\gamma}^{-1}}{1-N_{\gamma}^{-1}} .
\end{aligned}
$$

Note that $H_{\epsilon}(T)$ is an approximation of $H(T)$, and that for any $\epsilon>0$, ( $\operatorname{Ran} 84$, p. 298])

$$
H_{\epsilon}(T-\epsilon) \leq H(T) \leq H_{\epsilon}(T+\epsilon) .
$$

An application of the Selberg trace formula yields

$$
\begin{aligned}
\sum_{\lambda_{n} \leq \frac{1}{4}} h_{T}^{\epsilon}\left(r_{n}\right)+\sum_{\lambda_{n}>\frac{1}{4}} h_{T}^{\epsilon}\left(r_{n}\right)+\frac{1}{4 \pi} \int_{\mathbb{R}} h_{T}^{\epsilon}(r) \frac{-\phi^{\prime}}{\phi}\left(\frac{1}{2}+i r\right) d r \\
=\frac{|\mathcal{F}|}{4 \pi} \int_{\mathbb{R}} r h_{T}^{\epsilon}(r) \tanh (\pi r) d r \\
\quad+H_{\epsilon}(T)+\sum_{\{R\}} \frac{1}{2 m_{R} \sin \theta_{R}} \int_{\mathbb{R}} \frac{e^{-2 r \theta_{R}}}{1+e^{-2 \pi r}} h_{T}^{\epsilon}(r) d r+\frac{h_{T}^{\epsilon}(0)}{4} \operatorname{tr}\left(I-\mathfrak{S}\left(\frac{1}{2}\right)\right) \\
\quad-\tau g_{T}^{\epsilon}(0) \log 2-\frac{\tau}{2 \pi} \int_{\mathbb{R}} h_{T}^{\epsilon}(r) \psi(1+i r) d r .
\end{aligned}
$$

Our next goal is to let $\epsilon=e^{-T}$, and estimate each term, in terms of the variable $T$.

Recall from Theorem 3.3 that

$$
\mu(r) \leq C\left(r^{2}+\frac{1}{4}\right) \quad(r \geq 0) .
$$


Lemma 4.5. Let $\epsilon=e^{-T / 4}$,

$$
C_{13}=\frac{41}{6} C C_{10}
$$

Then

$$
\left|\sum_{\lambda_{n}>\frac{1}{4}} h_{T}^{\epsilon}\left(r_{n}\right)+\frac{1}{4 \pi} \int_{\mathbb{R}} h_{T}^{\epsilon}(r) \frac{-\phi^{\prime}}{\phi}\left(\frac{1}{2}+i r\right) d r\right|=\mathcal{O}_{13}\left(e^{3 T / 4}\right)
$$

Proof.

$$
\begin{aligned}
& \left|\sum_{\lambda_{n}>\frac{1}{4}} h_{T}^{\epsilon}\left(r_{n}\right)+\frac{1}{4 \pi} \int_{\mathbb{R}} h_{T}^{\epsilon}(r) \frac{-\phi^{\prime}}{\phi}\left(\frac{1}{2}+i r\right) d r\right| \\
& \quad \leq \sum_{\lambda_{n}>\frac{1}{4}}\left|h_{T}^{\epsilon}\left(r_{n}\right)\right|+\frac{1}{4 \pi} \int_{\mathbb{R}}\left|h_{T}^{\epsilon}(r)\right|\left|\frac{\phi^{\prime}}{\phi}\left(\frac{1}{2}+i r\right)\right| d r=\int_{0}^{\infty}\left|h_{T}^{\epsilon}(r)\right| d \mu(r) .
\end{aligned}
$$

By Theorem $3.3 \mu(r) \leq C\left(r^{2}+\frac{1}{4}\right)$. The result now follows from Lemma 4.3

Lemma 4.6. Let $\epsilon=e^{-T / 4}$,

$$
C_{14}=C_{10} \frac{296 \tau}{3 \pi}+C_{10} \frac{\tau}{2}+2 \tau \log 2
$$

then

$$
\frac{\tau}{2 \pi} \int_{\mathbb{R}}\left|h_{T}^{\epsilon}(r) \psi(1+i r)\right| d r+\left|\frac{h_{T}^{\epsilon}(0)}{4} \operatorname{tr}\left(I-\mathfrak{S}\left(\frac{1}{2}\right)\right)\right|+\left|\tau g_{T}^{\epsilon}(0) \log 2\right|=\mathcal{O}_{14}\left(e^{3 T / 4}\right) .
$$

Proof. We previously saw (equation (2.2) ) that for $r \in \mathbb{R}$,

$$
|\psi(1+i r)| \leq 4|r|^{1 / 4}+4
$$

Hence $|\psi(1+i r)| \leq 8(1+|r|)$, and

$$
\frac{\tau}{2 \pi} \int_{-\infty}^{\infty}\left|h_{T}^{\epsilon}(r) \psi(1+i r)\right| d r \leq \frac{\tau}{2 \pi} \int_{-\infty}^{\infty}\left|h_{T}^{\epsilon}(r)\right| 8(1+|r|) d r=\frac{\tau}{2 \pi} \int_{0}^{\infty}\left|h_{T}^{\epsilon}(r)\right| d u(r),
$$

where

$$
u(r)=\int_{-r}^{r} 8(1+|x|) d x=16\left(\frac{r^{2}}{2}+r\right) \leq 16\left(\frac{3}{2} r^{2}+1\right) \quad(r \in \mathbb{R}) .
$$

An application of Lemma 4.3 yields

$$
\frac{\tau}{2 \pi} \int_{0}^{\infty}\left|h_{T}^{\epsilon}(r)\right| d u(r) \leq C_{10} \frac{296 \tau}{3 \pi} \frac{e^{T / 2}}{\epsilon}
$$

Next, an application of Lemma 4.2 the inequalities $\left|\operatorname{tr}\left(I-\mathfrak{S}\left(\frac{1}{2}\right)\right)\right| \leq 2 \tau$ and $\left|g_{T}^{\epsilon}(0)\right| \leq 2$, imply

$$
\begin{aligned}
\left|\frac{h_{T}^{\epsilon}(0)}{4} \operatorname{tr}\left(I-\mathfrak{S}\left(\frac{1}{2}\right)\right)\right|+\left|\tau g_{T}^{\epsilon}(0) \log 2\right| & \leq \frac{\tau C_{10}}{2} e^{T / 2}+2 \tau \log 2 \\
& \leq\left(\frac{\tau C_{10}}{2}+2 \tau \log 2\right) e^{3 T / 4}
\end{aligned}
$$


Lemma 4.7. Let $\epsilon=e^{-T / 4}$,

$$
C_{15}=\frac{56 C_{10}}{3}\left|\sum_{\{R\}} \frac{1}{2 m_{R} \sin \theta_{R}}\right|,
$$

then

$$
\left|\sum_{\{R\}} \frac{1}{2 m_{R} \sin \theta_{R}} \int_{\mathbb{R}} \frac{e^{-2 r \theta_{R}}}{1+e^{-2 \pi r}} h_{T}^{\epsilon}(r) d r\right|=\mathcal{O}_{15}\left(e^{3 T / 4}\right) .
$$

Proof. Since

$$
\begin{gathered}
\left|\frac{e^{-2 r \theta_{R}}}{1+e^{-2 \pi r}}\right| \leq 1, \\
\left|\sum_{\{R\}} \frac{1}{2 m_{R} \sin \theta_{R}} \int_{\mathbb{R}} \frac{e^{-2 r \theta_{R}}}{1+e^{-2 \pi r}} h_{T}^{\epsilon}(r) d r\right| \leq\left|\sum_{\{R\}} \frac{1}{2 m_{R} \sin \theta_{R}}\right| \int_{0}^{\infty}\left|h_{T}^{\epsilon}(r)\right| d u(r),
\end{gathered}
$$

where $u(r)=2 r$. Hence $|u(r)| \leq 2 r^{2}+2$, and applying Lemma 4.3 yields

$$
\left|\sum_{\{R\}} \frac{1}{2 m_{R} \sin \theta_{R}} \int_{\mathbb{R}} \frac{e^{-2 r \theta_{R}}}{1+e^{-2 \pi r}} h_{T}^{\epsilon}(r) d r\right| \leq\left(\frac{56 C_{10}}{3}\left|\sum_{\{R\}} \frac{1}{2 m_{R} \sin \theta_{R}}\right|\right) e^{3 T / 4} .
$$

\section{Lemma 4.8.}

$$
\frac{|\mathcal{F}|}{4 \pi} \int_{-\infty}^{\infty}\left|h_{T}^{\epsilon}(r) r \tanh (\pi r)\right| d r \leq \frac{3}{2 \pi}|\mathcal{F}| C_{10} \frac{e^{T / 2}}{\epsilon} .
$$

Proof. Since $r \tanh (\pi r)$ is even, it suffices to estimate

$$
\frac{|\mathcal{F}|}{2 \pi} \int_{0}^{\infty}\left|h_{T}^{\epsilon}(r) r \tanh (\pi r)\right| d r \leq \frac{|\mathcal{F}|}{2 \pi} \int_{0}^{\infty}\left|h_{T}^{\epsilon}(r)\right| r d r=\frac{|\mathcal{F}|}{2 \pi} \int_{0}^{\infty}\left|h_{T}^{\epsilon}(r)\right| d u(r),
$$

where $u(r)=\int_{0}^{r} x d x=\frac{r^{2}}{2}$. (Note that we used the trivial estimate $|\tanh (\pi r)| \leq 1$.) The result now follows from Lemma 4.3.

Our main result for this section is

Lemma 4.9. Let $\epsilon=e^{-T / 4}$, and let

$$
C_{16}=C_{12}+C_{13}+C_{14}+C_{15}+\frac{3}{2 \pi}|\mathcal{F}| C_{10} .
$$

Then

$$
H_{\epsilon}(T)=\sum_{\lambda_{k} \leq \frac{1}{4}} \frac{e^{T s_{k}}}{s_{k}}+\mathcal{O}_{16}\left(e^{3 T / 4}\right) .
$$

Proof. The proof follows immediately from equation (4.3), and Lemmas 4.4, 4.5, 4.6. $4.7,4.8$, 
4.5. Upper bound for the Huber constant. In this section we prove the main result of this paper.

4.6. Asymptotics for $H(T)$.

Lemma 4.10. Let $\epsilon=e^{-T / 4}$,

$$
\begin{aligned}
& C_{17}=4 \mathcal{A}+4 C_{16}, \\
& C_{18}=4 \mathcal{A}+5 C_{16} .
\end{aligned}
$$

Then

(1) $H(T)-H_{\epsilon}(T)=\mathcal{O}_{17}\left(e^{3 T / 4}\right)$.

(2) $H(T)=\sum_{\lambda_{k} \leq \frac{1}{4}} \frac{e^{T s_{k}}}{s_{k}}+\mathcal{O}_{18}\left(e^{3 T / 4}\right)$.

Proof. We first prove (1). Recall from equation (4.2) that

$$
H_{\epsilon}(T-\epsilon) \leq H(T) \leq H_{\epsilon}(T+\epsilon) \text {. }
$$

In addition, it follows from the definition of $H_{\epsilon}(T)$ that

$$
H_{\epsilon}(T-\epsilon) \leq H_{\epsilon}(T) \leq H_{\epsilon}(T+\epsilon) \text {. }
$$

By Lemma 4.9 we have

$$
H_{\epsilon}(T \pm \epsilon)=\sum_{\lambda_{k} \leq \frac{1}{4}} \frac{e^{(T \pm \epsilon) s_{k}}}{s_{k}}+\mathcal{O}_{16}\left(e^{3(T \pm \epsilon) / 4}\right) .
$$

Now, setting $\epsilon=e^{-T / 4}$, it follows from elementary estimates that

$$
\left|H_{\epsilon}(T+\epsilon)-H_{\epsilon}(T-\epsilon)\right| \leq 4 \mathcal{A} e^{3 T / 4}+4 C_{16} e^{3 T / 4} .
$$

Part (1) now follows from (4.4) and (4.5). Part (2) follows immediately from Lemma 4.9

\subsection{The Chebyshev function. Define}

$$
\Psi(T) \equiv \sum_{l(\gamma) \leq T} \Lambda(\gamma)
$$

where $\Lambda(\gamma)=l\left(\gamma_{0}\right)$. Since $\Gamma$ is discrete, there exists a hyperbolic conjugacy class $\left\{P_{s}\right\}$ with minimal norm $N\left(P_{s}\right)>1$. Choose $c>0$ so that

$$
1<c<N\left(P_{s}\right) \text {. }
$$

Lemma 4.11. Let

$$
C_{19}=C_{18}+\frac{4}{1-c^{-1}}\left(2 \mathcal{A}+C_{18}\right) .
$$

Then

$$
\Psi(T)=\sum_{\lambda_{k} \leq \frac{1}{4}} \frac{e^{T s_{k}}}{s_{k}}+\mathcal{O}_{19}\left(e^{3 T / 4}\right) .
$$

Proof. By Lemma 4.10.

$$
H(T)=\sum_{\lambda_{k} \leq \frac{1}{4}} \frac{e^{T s_{k}}}{s_{k}}+\mathcal{O}_{18}\left(e^{3 T / 4}\right) .
$$

Since

$$
H(T)=\sum_{l(\gamma) \leq T} \Lambda(\gamma) \frac{1+N_{\gamma}^{-1}}{1-N_{\gamma}^{-1}},
$$


we can rewrite $H(T)$ as

$$
H(T)=\Psi(T)+\sum_{l(\gamma) \leq T} \Lambda(\gamma) \frac{2 N_{\gamma}^{-1}}{1-N_{\gamma}^{-1}} .
$$

Both of the above terms are positive for all $T$. Thus, from Lemma 4.10 and a crude estimate (for each small eigenvalue, $\frac{1}{2} \leq s_{k} \leq 1$, hence $e^{s_{k} t} \leq e^{t}$ ), it follows that

$$
\Psi(T) \leq H(T) \leq\left(2 \mathcal{A}+C_{18}\right) e^{T} .
$$

But

$$
\begin{aligned}
\sum_{l(\gamma) \leq T} \Lambda(\gamma) \frac{2 N_{\gamma}^{-1}}{1-N_{\gamma}^{-1}} \leq \frac{2}{1-c^{-1}} \sum_{l(\gamma) \leq T} \Lambda(\gamma) N_{\gamma}^{-1} & =\frac{2}{1-c^{-1}} \int_{0}^{T} e^{-x} d \Psi(x) \\
& \leq \frac{2}{1-c^{-1}}\left(2 \mathcal{A}+C_{18}\right)(1+T) .
\end{aligned}
$$

The last inequality is obtained by integrating by parts and using (4.6). Next, using the crude bound $(1+T) \leq 2 e^{3 T / 4}$, we obtain

$$
\sum_{l(\gamma) \leq T} \Lambda(\gamma) \frac{2 N_{\gamma}^{-1}}{1-N_{\gamma}^{-1}} \leq \frac{4}{1-c^{-1}}\left(2 \mathcal{A}+C_{18}\right) e^{3 T / 4} .
$$

Finally,

$$
\left|\Psi(T)-\sum_{s_{k}} \frac{e^{T s_{k}}}{s_{k}}\right| \leq\left(C_{18}+\frac{4}{1-c^{-1}}\left(2 \mathcal{A}+C_{18}\right)\right) e^{3 T / 4} .
$$

\subsection{The Huber constant. Let}

$$
\Theta(T)=\sum_{\substack{l(\gamma) \leq T \\ \gamma \text { primitive }}} \Lambda(\gamma)
$$

Choose $\mu>0$ so that $\mu<l(\gamma)$ for all $\gamma \in \mathcal{C}(M)$.

Lemma 4.12 .

$$
\Theta(T)=\sum_{\lambda_{k} \leq \frac{1}{4}} \frac{e^{T s_{k}}}{s_{k}}+\mathcal{O}_{20}\left(e^{3 T / 4}\right)
$$

where

$$
C_{20}=C_{19}+\frac{4}{\mu}\left(2 \mathcal{A}+C_{18}\right) .
$$

Proof. Let $m(T)=[T / \mu]$, then ([Bus92, p. 251])

$$
\Psi(T)-\Theta(T)=\sum_{m=2}^{m(T)} \Theta(T / m) \leq m(T) \Theta(T / 2) \leq \frac{T}{\mu} \Psi(T / 2) .
$$

By (4.6),

Hence,

$$
\frac{T}{\mu} \Psi(T / 2) \leq \frac{1}{\mu} T e^{T / 2}\left(2 \mathcal{A}+C_{18}\right) \leq \frac{4}{\mu} e^{3 T / 4}\left(2 \mathcal{A}+C_{18}\right) .
$$

$$
|\Theta(T)-\Psi(T)| \leq \frac{4}{\mu} e^{3 T / 4}\left(2 \mathcal{A}+C_{18}\right) .
$$

The lemma now follows. 
Let $x=e^{T}$, and let

$$
\theta(x) \equiv \sum_{\substack{N_{\gamma} \leq x \\ \gamma \text { primitive }}} \Lambda(\gamma) .
$$

With this change of variable, Lemma 4.12 becomes

$$
\theta(x)=\sum_{\lambda_{k} \leq \frac{1}{4}} \frac{x^{s_{k}}}{s_{k}}+\mathcal{O}_{20}\left(x^{3 / 4}\right) .
$$

Let

$$
\operatorname{li}(x)=\int_{2}^{x} \frac{d y}{\log y}
$$

and recall the definition of the constant $c$ (4.7). We have

Lemma 4.13. Let

$$
C_{21}=|c-2| \frac{1}{\log 2}+|2-\sqrt{c}| \frac{2}{\log c} .
$$

Then for $s \in\left[\frac{1}{2}, 1\right]$,

$$
\left|\int_{c}^{x} \frac{y^{s-1}}{\log y} d y-\operatorname{li}\left(x^{s}\right)\right| \leq C_{21} .
$$

Proof. An elementary argument shows that

$$
\left|\int_{c}^{x} \frac{y^{s-1}}{\log y} d y-\operatorname{li}\left(x^{s}\right)\right|=\left|\int_{c^{s}}^{2} \frac{d y}{\log y}\right| .
$$

Now, if $c^{s} \geq 2$, then

$$
\left|\int_{c^{s}}^{2} \frac{d y}{\log y}\right| \leq\left(c^{s}-2\right) \frac{1}{\log 2} \leq(c-2) \frac{1}{\log 2}
$$

If $c^{s}<2$, then

$$
\left|\int_{c^{s}}^{2} \frac{d y}{\log y}\right| \leq\left(2-c^{s}\right) \frac{1}{\log c^{s}} \leq\left(2-c^{1 / 2}\right) \frac{2}{\log c} .
$$

Hence, in either case,

$$
\left|\int_{c^{s}}^{2} \frac{d y}{\log y}\right| \leq|c-2| \frac{1}{\log 2}+\left|2-c^{1 / 2}\right| \frac{2}{\log c} .
$$

Lemma 4.14. Let

$$
C_{22}=\left(1-\frac{1}{\log 2}\right)^{-1}
$$

Then for $x>1$,

$$
\operatorname{li}(x) \leq C_{22} \frac{x}{\log x} .
$$

Proof. Integrating by parts give us

$$
\operatorname{li}(x)=\int_{2}^{x} \frac{d y}{\log y} \leq \frac{x}{\log x}+\int_{2}^{x} \frac{d y}{(\log y)^{2}} \leq \frac{x}{\log x}+\frac{1}{\log 2} \int_{2}^{x} \frac{d y}{\log y} .
$$

The lemma now follows. 
Theorem 4.15. Let

$$
C_{u}=C_{21} \mathcal{A}+C_{20} \frac{c^{3 / 4}}{\log c}+C_{20}+C_{20} C_{22}+\frac{3}{4} C_{20} C_{21} .
$$

Then for all $x>1$,

In particular, $C_{M} \leq C_{u}$.

$$
\left|\pi(x)-\sum_{\lambda_{k} \leq \frac{1}{4}} \operatorname{li}\left(x^{s_{k}}\right)\right| \leq C_{u} \frac{x^{3 / 4}}{\log x} .
$$

Proof. First note that

$$
\pi(x)=\int_{c}^{x} \frac{d \theta(y)}{\log y} .
$$

First, for notational convenience, set

$$
f(y) \equiv \theta(y)-\sum_{\lambda_{k} \leq \frac{1}{4}} \frac{y^{s_{k}}}{s_{k}}=\mathcal{O}_{20}\left(y^{3 / 4}\right) .
$$

Next,

$$
\int_{c}^{x} \frac{d \theta(y)}{\log y}=\sum_{\lambda_{k} \leq \frac{1}{4}} \int_{c}^{x} \frac{y^{s_{k}-1}}{\log y} d y+\int_{c}^{x} \frac{d f(y)}{\log y} .
$$

By Lemma 4.13 ,

$$
\sum_{\lambda_{k} \leq \frac{1}{4}} \int_{c}^{x} \frac{y^{s_{k}-1}}{\log y} d y=\sum_{\lambda_{k} \leq \frac{1}{4}} \operatorname{li}\left(x^{s_{k}}\right)+\mathcal{A} \mathcal{O}_{21}(1)
$$

Now

$$
\int_{c}^{x} \frac{d f(y)}{\log y}=\frac{f(x)}{\log x}-\frac{f(c)}{\log c}+\int_{c}^{x} f(y) \frac{d y}{y(\log y)^{2}},
$$

and (using Lemma 4.13),

$$
\begin{aligned}
\int_{c}^{x}|f(y)| \frac{d y}{y(\log y)^{2}} & \leq C_{20} \int_{c}^{x} y^{3 / 4} \frac{d y}{y(\log y)^{2}} \\
& =C_{20}\left(\frac{c^{3 / 4}}{\log c}+\frac{x^{3 / 4}}{\log x}+\frac{3}{4} \int_{c}^{x} y^{\left(\frac{3}{4}-1\right)} \frac{d y}{\log y}\right) \\
& \leq C_{20}\left(\frac{c^{3 / 4}}{\log c}+\frac{x^{3 / 4}}{\log x}+\frac{3}{4}\left(\operatorname{li}\left(x^{3 / 4}\right)+C_{21}\right)\right) .
\end{aligned}
$$

But by Lemma 4.14

$$
\frac{3}{4} \operatorname{li}\left(x^{3 / 4}\right) \leq C_{22} \frac{x^{3 / 4}}{\log x} .
$$

Hence, putting everything together, for $x>1$,

$$
\left|\pi(x)-\sum_{\lambda_{k} \leq \frac{1}{4}} \operatorname{li}\left(x^{s_{k}}\right)\right| \leq C_{21} \mathcal{A}+C_{20} \frac{c^{3 / 4}}{\log c}+C_{20} \frac{x^{3 / 4}}{\log x}+C_{20} C_{22} \frac{x^{3 / 4}}{\log x}+\frac{3}{4} C_{20} C_{21},
$$

and using the trivial inequality $1<\frac{x^{3 / 4}}{\log x}$, we obtain

$$
\left|\pi(x)-\sum_{\lambda_{k} \leq \frac{1}{4}} \operatorname{li}\left(x^{s_{k}}\right)\right| \leq C_{u} \frac{x^{3 / 4}}{\log x}
$$


where

$$
C_{u}=C_{21} \mathcal{A}+C_{20} \frac{c^{3 / 4}}{\log c}+C_{20}+C_{20} C_{22}+\frac{3}{4} C_{20} C_{21}
$$

4.9. The Huber constant for $\operatorname{PSL}(2, \mathbb{Z})$. For $\Gamma=\operatorname{PSL}(2, \mathbb{Z})$, we can take $c=$ 6.85. Also, $\Gamma$ has no small eigenvalues (except for the trivial one), so $\mathcal{A}=1$. Using the computations in 3.2 , an explicit value for $C_{u}$ can be calculated:

Theorem 4.16. Let $\Gamma=\operatorname{PSL}(2, \mathbb{Z})$. Then $C_{u}=16,607,349,020,658$ is an upper bound for the Huber constant.

Using the theory laid out in Sar82, and the excellent open-source computational program PARI/GP The05, we can explicitly calculate (and list out) the length spectrum for $\operatorname{PSL}(2, \mathbb{Z})$. Our computations suggest that

$$
C_{M}<2 .
$$

So it seems that our result is probably not sharp!

\section{COMPUTER EXPERIMENTS FOR PSL $(2, \mathbb{Z})$}

Let $\Gamma_{0}=\operatorname{PSL}(2, \mathbb{Z})$, and $\Gamma$ be a finite index subgroup of $\Gamma_{0}$. Let $\left\{P_{0}\right\}$ denote the set of primitive hyperbolic conjugacy classes of $\Gamma$. We compute length spectrum (norms of primitive hyperbolic conjugacy classes) using the ideas of Sar82.

Let $Q(x, y)=a x^{2}+b x y+c y^{2}$ be a primitive, indefinite binary quadratic form of discriminant $d=b^{2}-4 a c>0$. Two forms $[a, b, c]$ and $\left[a^{\prime}, b^{\prime}, c^{\prime}\right]$ are called equivalent (in the narrow sense) if they are related by a unimodular transformation.

Consider the map $\phi$ which sends the form $[a, b, c]$ to the matrix

$$
\left(\begin{array}{cc}
\frac{t_{d}-b u_{d}}{2} & -c u_{d} \\
a u_{d} & \frac{t_{d}+b u_{d}}{2}
\end{array}\right)
$$

where $t_{d}, u_{d}>0$ is the fundamental solutions of Pell's equation $t^{2}-d u^{2}=4$. Set

$$
\epsilon_{d}=\frac{t_{d}+\sqrt{d} u_{d}}{2}
$$

Let $\mathcal{D}$ be the set of fundamental discriminants $\{d>0, d \equiv 0,1 \bmod 4\}$ and let $h(d)$ denote the number of inequivalent primitive forms of discriminant $d$. For each $d \in \mathcal{D}$ let $Q_{1}(d), \ldots, Q_{h(d)}(d)$ be a complete set of inequivalent forms of discriminate $d$. Finally, set

$$
Q F=\bigcup_{d \in \mathcal{D}}\left\{Q_{1}(d), \ldots Q_{h(d)}(d)\right\}
$$

Lemma 4.17 ([Sar82]). The set $\phi(Q F)$ is the set of conjugacy classes of primitive hyperbolic elements of $\operatorname{PSL}(2, \mathbb{Z})$. The norm of each hyperbolic element $\phi\left(Q_{i}(d)\right)$ is $\epsilon_{d}^{2}$. 
Using the above ideas, one can compute the primitive conjugacy classes for $\Gamma_{0}=\operatorname{PSL}(2, \mathbb{Z})$. We next explain how to compute the length spectrum for $\Gamma$, a finite-index normal subgroup of $\Gamma_{0}$.

Let $\Gamma \triangleleft \Gamma_{0}$ be a finite-index normal subgroup of $\Gamma_{0}=\operatorname{PSL}(2, \mathbb{Z}), G=\Gamma_{0} / \Gamma$, and $n=|G|$. For each primitive conjugacy class $\left\{P_{\gamma}\right\}$ of $\Gamma_{0}$ let $m$ represent the order of $P_{\gamma} \in G$. It follows that $\left\{P_{\gamma}^{m}\right\}$ is a subset of $\Gamma$ that splits into conjugacy classes with respect to $\Gamma$.

Lemma 4.18 ([Sar82]). The number of conjugacy classes into which $\left\{P_{\gamma}^{m}\right\}$ splits in $\Gamma$ is $k=n / m=|G| / m$.

\section{Algorithm FOR COMPUTING PRIMItIVE CONJUGACY CLASSES}

Let $\Gamma \triangleleft \Gamma_{0}, x>0$. We wish to compute the norms and multiplicities of all primitive hyperbolic conjugacy classes $\left\{P_{\gamma}\right\}$ with norm $N\left(\left\{P_{\gamma}\right\}\right) \leq x$.

Step 1. Compute the primitive conjugacy classes for the modular group $\Gamma_{0}$ of norm $x$ or less. Since $\epsilon_{d}^{2}$ is the norm of a prime element that is mapped by the function $\phi$ it follows that $\sqrt{x} \geq \epsilon_{d}>\sqrt{d} / 2$. Thus it suffices to consider discriminants $d<4 x$ in order to guarantee that all norms less than $x$ are achieved. In other words, if $d>4 x$, then $\epsilon_{d}^{2}>x$.

For each $d \in \mathcal{D}_{x}=\left\{d \in \mathcal{D} \mid \epsilon_{d}^{2} \leq x\right\}$, compute $h(d)$ and representatives of inequivalent quadratic forms of discriminant $d$. For each representative $Q_{i}(d)$ compute $\phi\left(Q_{i}(d)\right)$ in order to obtain representatives for $\left\{P_{\gamma}\right\}$ in $\Gamma_{0}$

Step 2. From step 1, let $z=P_{\gamma}$ be a representative of a class in $\Gamma_{0}$ of norm $\epsilon_{d}^{2}$. Compute the order $m$ of $z$ in $G=\Gamma_{0} / \Gamma$. Then $\left\{P_{\gamma}^{m}\right\}$ splits into $k=|G| / m$ primitive conjugacy classes each with norm $\epsilon_{d}^{2 m}$.

Step 3. From step 2, we count the primitive conjugacy classes with norm $\epsilon_{d}^{2 m} \leq x$.

One thing we can do is verify the prime geodesic theorem for the group $\Gamma$. We can implement these algorithms using the computer package PARI/GP.

\section{Pari/GP Algorithm.}

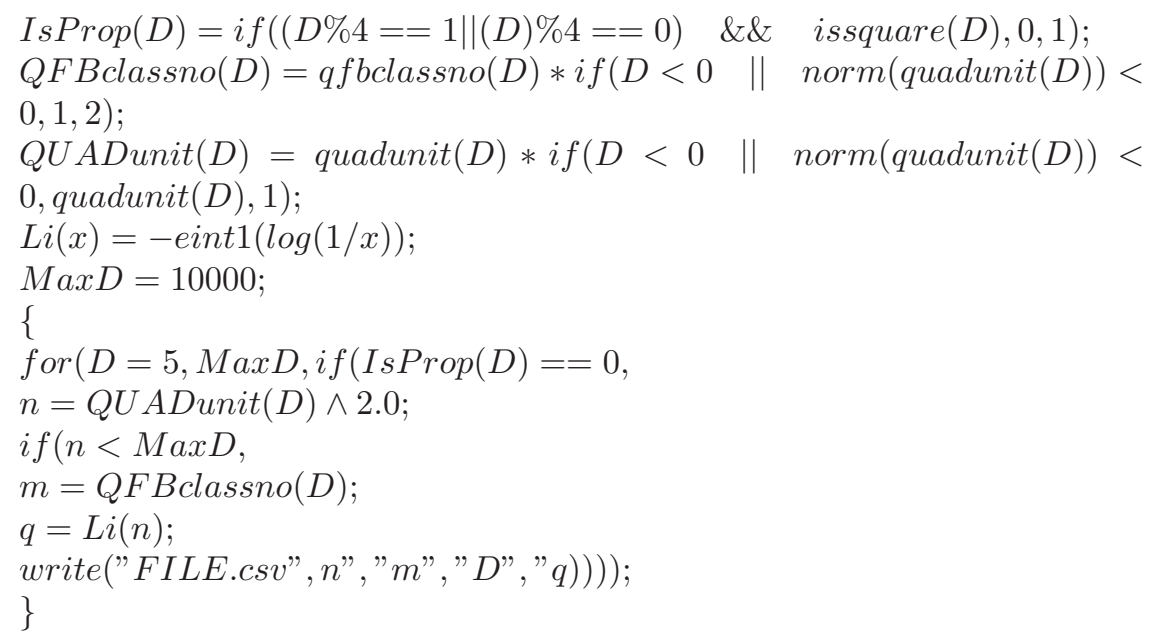


TABle 4. Data for the modular group

\begin{tabular}{|l|l|l|l|l|l|l|l|l|l|l|}
\hline 1 & 2 & 3 & 4 & 5 & 6 & 7 & 8 & 9 & 10 & 11 \\
\hline 6.85 & 1 & 5 & 4.68 & 1 & 4.68 & 3.68 & 3.05 & 1.21 & 2.62 & 1.41 \\
\hline 13.93 & 2 & 12 & 7.75 & 3 & 2.58 & 4.75 & 4.44 & 1.07 & 3.73 & 1.27 \\
\hline 22.96 & 2 & 21 & 10.87 & 5 & 2.17 & 5.87 & 5.92 & .99 & 4.79 & 1.23 \\
\hline 33.97 & 2 & 32 & 14.17 & 7 & 2.02 & 7.17 & 7.49 & .96 & 5.83 & 1.23 \\
\hline 33.97 & 1 & 8 & 14.17 & 8 & 1.77 & 6.17 & 7.49 & .82 & 5.83 & 1.06 \\
\hline 46.98 & 2 & 45 & 17.69 & 10 & 1.77 & 7.69 & 9.15 & .84 & 6.85 & 1.12 \\
\hline 61.98 & 4 & 60 & 21.45 & 14 & 1.53 & 7.45 & 10.87 & .68 & 7.87 & 0.95 \\
\hline 78.99 & 2 & 77 & 25.45 & 16 & 1.59 & 9.45 & 12.68 & .75 & 8.89 & 1.06 \\
\hline 97.99 & 2 & 24 & 29.69 & 18 & 1.65 & 11.69 & 14.55 & .80 & 9.9 & 1.18 \\
\hline 97.99 & 4 & 96 & 29.69 & 22 & 1.35 & 7.69 & 14.55 & .53 & 9.9 & 0.78 \\
\hline 118.99 & 1 & 13 & 34.17 & 23 & 1.49 & 11.17 & 16.48 & .68 & 10.91 & 1.02 \\
\hline 118.99 & 2 & 117 & 34.17 & 25 & 1.37 & 9.17 & 16.48 & .56 & 10.91 & 0.84 \\
\hline 141.99 & 4 & 140 & 38.9 & 29 & 1.34 & 9.9 & 18.48 & .54 & 11.92 & 0.83 \\
\hline 166.99 & 4 & 165 & 43.86 & 33 & 1.33 & 10.86 & 20.53 & .53 & 12.92 & 0.84 \\
\hline 193.99 & 2 & 48 & 49.06 & 35 & 1.40 & 14.06 & 22.65 & .62 & 13.93 & 1.01 \\
\hline 193.99 & 4 & 192 & 49.06 & 39 & 1.26 & 10.06 & 22.65 & .44 & 13.93 & 0.72 \\
\hline 223 & 4 & 221 & 54.49 & 43 & 1.27 & 11.49 & 24.82 & .46 & 14.93 & 0.77 \\
\hline 254 & 2 & 28 & 60.15 & 45 & 1.34 & 15.15 & 27.04 & .56 & 15.94 & 0.95 \\
\hline 254 & 4 & 252 & 60.15 & 49 & 1.23 & 11.15 & 27.04 & .41 & 15.94 & 0.7 \\
\hline 287 & 4 & 285 & 66.04 & 53 & 1.25 & 13.04 & 29.31 & .45 & 16.94 & 0.77 \\
\hline 322 & 2 & 80 & 72.17 & 55 & 1.31 & 17.17 & 31.63 & .54 & 17.94 & 0.96 \\
\hline 322 & 1 & 20 & 72.17 & 56 & 1.29 & 16.17 & 31.63 & .51 & 17.94 & 0.9 \\
\hline 322 & 4 & 320 & 72.17 & 60 & 1.20 & 12.17 & 31.63 & .38 & 17.94 & 0.68 \\
\hline 359 & 4 & 357 & 78.51 & 64 & 1.23 & 14.51 & 34 & .43 & 18.95 & 0.77 \\
\hline 398 & 2 & 44 & 85.08 & 66 & 1.29 & 19.08 & 36.42 & .52 & 19.95 & 0.96 \\
\hline 398 & 8 & 396 & 85.08 & 74 & 1.15 & 11.08 & 36.42 & .30 & 19.95 & 0.56 \\
\hline 439 & 2 & 437 & 91.88 & 76 & 1.21 & 15.88 & 38.88 & .41 & 20.95 & 0.76 \\
\hline 482 & 4 & 120 & 98.89 & 80 & 1.24 & 18.89 & 41.39 & .46 & 21.95 & 0.86 \\
\hline 482 & 8 & 480 & 98.89 & 88 & 1.12 & 10.89 & 41.39 & .26 & 21.95 & 0.5 \\
\hline 527 & 4 & 525 & 106.12 & 92 & 1.15 & 14.12 & 43.94 & .32 & 22.96 & 0.62 \\
\hline 574 & 4 & 572 & 113.57 & 96 & 1.18 & 17.57 & 46.53 & .38 & 23.96 & 0.73 \\
\hline 623 & 2 & 69 & 121.23 & 98 & 1.24 & 23.23 & 49.16 & .47 & 24.96 & 0.93 \\
\hline & & & & & & & & & & \\
\hline
\end{tabular}

Key: Length spectrum calculations for the modular group.

(1) $x$, the actual primitive length spectrum (norms); when norms repeat, they come from different quadratic forms,

(2) multiplicity of given norm,

(3) discriminant of corresponding quadratic form,

(4) $\operatorname{li}(x)$, the integral logarithm of $x$ where $x$ is equal to the value of column 1 ,

(5) $\pi(x)$, a running total of the number of norms less than $x$ where $x$ is equal to the value of column 1 ,

(6) $\frac{\pi(x)}{\mathrm{li}(x)}$; this column verifies the prime geodesic theorem,

(7) $|\operatorname{li}(x)-\pi(x)|$,

(8) $x^{3 / 4} /(\log (x))^{1 / 2}$,

(9) the ratio of $(7) /(8)$, 
(10) $x^{1 / 2}$, this is the conjectured true error term,

(11) the ratio of $(7) /(10)$.

\section{REFERENCES}

[Bo07] Bosman, J.: A polynomial with Galois group $\mathrm{SL}_{2}\left(\mathbb{F}_{16}\right)$. LMS J. Comput. Math. 10 (2007), 1461-1570. MR2365691(2008k:12008)

[BKK05] Burgos Gil, J. I.; Kramer, J.; Kühn, U.: Arithmetic characteristic classes of automorphic vector bundles. Doc. Math. 10 (2005), 619-716. MR2218402 (2007e:14039)

[BKK07] Burgos Gil, J. I.; Kramer, J.; Kühn, U.: Cohomological arithmetic Chow rings. J. Inst. Math. Jussieu 6 (2007), 1-172. MR2285241(2008f:14039)

[Bus92] Buser, P.: Geometry and spectra of compact Riemann surfaces, Progress in Mathematics, vol. 106, Birkhäuser Boston Inc., Boston, MA, 1992. MR 1183224 (93g:58149)

[CL09] Carls, R.; Lubicz, D.: A p-adic quasi-quadratic time point counting algorithm. Int. Math. Res. Not. (2009), no. 4, 698-735. MR2480098 (2010c:14020)

[Chl08] Chambert-Loir, A.: Compter (rapidement) le nombre de solutions d'équations dans les corps finis. Séminaire Bourbaki. Vol. 2006/2007. Astérisque no. 317 (2008), Exp. no. 968, vii, 39-90. MR2487730(2010a:11048)

[Cou09] Couveignes, J.-M.: Linearizing torsion classes in the Picard group of algebraic curves over finite fields. J. Algebra 321 (2009), 2085-2118. MR 2501511

[Edi06] Edixhoven, B.: On the computation of the coefficients of a modular form. Algorithmic number theory, 30-39, Lecture Notes in Comput. Sci., 4076, Springer, Berlin, (2006). MR 2282913 (2007k:11085)

[ECJMB] Edixhoven, B.; Couveignes, J.-M.; de Jong, R.; Merkl F.; Bosman, J.: On the computation of coefficients of a modular form, preprint, arxiv.org/abs/math/0605244. This version will be used as the basis for a book that will appear in the series Annals of Mathematics Studies of Princeton University Press. MR 2530982 (2010d:11001)

[EGM98] Elstrodt, J.; Grunewald, F.; Mennicke, J.: Groups acting on hyperbolic space, Springer Monographs in Mathematics, Springer-Verlag, Berlin, 1998, Harmonic analysis and number theory. MR1483315 (98g:11058)

[Fis87] Fischer, J.: An approach to the Selberg trace formula via the Selberg zeta-function, Lecture Notes in Mathematics, vol. 1253, Springer-Verlag, Berlin, 1987. MR892317 (88f:11053)

[FG09] Freixas i Montplet, G.: An arithmetic Riemann-Roch theorem for pointed stable curves. Ann. Sci. Éc. Norm. Supér. 42 (2009), 335-369. MR2518081

[GR65] Gradshteyn, I.S.; and Ryzhik, I.M: Table of integrals, series, and products, Fourth edition prepared by Ju. V. Geronimus and M. Ju. Ceĭtlin. Translated from the Russian by Scripta Technica, Inc. Translation edited by Alan Jeffrey, Academic Press, New York, 1965. MR0197789 (33:5942)

[Ha09] Hahn, T.: An arithmetic Riemann-Roch theorem for metrics with cusps. Ph.D. dissertation, Humboldt University, (2009).

[Hej76] Hejhal, D.A.: The Selberg trace formula for PSL $(2, R)$. Vol. I, Springer-Verlag, Berlin, 1976, Lecture Notes in Mathematics, vol. 548. MR0439755 (55:12641)

[Hej83] - The Selberg trace formula for $\operatorname{PSL}(2, \mathbf{R})$. Vol. 2, Lecture Notes in Mathematics, vol. 1001, Springer-Verlag, Berlin, 1983. MR711197 (86e:11040)

[Hub59] Huber, H.: Zur analytischen theorie hyperbolischer raumformen und bewegungsgruppen, Math. Ann. 138 (1959), 1-26. MR0109212 (22:99)

[Hub61a] _ Zur analytischen theorie hyperbolischer raumformen und bewegungsgruppen ii, Math. Ann. 142 (1960/1961), 385-398. MR0126549 (23:A3845)

[Hub61b] _ Nachtrag zu ii, Math. Ann. 143 (1961), 463-464. MR0154980 (27:4923)

[Iwa97] Iwaniec, H.: Topics in classical automorphic forms, Graduate Studies in Mathematics, vol. 17, American Mathematical Society, Providence, RI, 1997. MR1474964(98e:11051)

[Iwa02] _ Spectral methods of automorphic forms, second ed., Graduate Studies in Mathematics, vol. 53, American Mathematical Society, Providence, RI, 2002. MR1942691 (2003k:11085)

[JK01] Jorgenson, J.; Kramer, J.: Bounds for special values of Selberg zeta functions of Riemann surfaces, J. Reine Angew. Math. 541 (2001), 1-28. MR1876283 (2002k:11145) 
[JK02] - On the error term of the prime geodesic theorem, Forum Math. 14 (2002), no. 6, 901-913. MR 1932525 (2003h:11059)

[JK06] - Bounds on canonical Green's functions, Compos. Math. 142 (2006), no. 3, 679-700. MR2231197

[JK09] - Bounds on Faltings's delta function through covers, Annals of Math. 170 (2009), 1-43. MR2521110(2010g:14031)

[La06] Lauder, A.: A recursive method for computing zeta functions of varieties. LMS J. Comput. Math. 9 (2006), 222-269. MR2261044(2007g:14022)

[Ran78] Randol, B.: The Riemann hypothesis for Selberg's zeta-function and the asymptotic behavior of the eigenvalues of the Laplace operator, Trans. Amer. Math. Soc. 236 (1978), 209-223. MR0472728 (57:12420)

[Ran84] Chapter xi: Eigenvalues in Riemannian geometry, Pure and Applied Mathematics (I. Chavel), vol. 115, Academic Press Inc., Orlando, FL, 1984, Including a chapter by Burton Randol, With an appendix by Jozef Dodziuk. MR768584 (86g:58140)

[Sar80] Sarnak, P.: Prime geodesic theorems, Ph.D. thesis, Stanford University, 1980.

[Sar82] Class numbers of binary quadratic forms, J. Number Theory 15 (1982), no. 1, 226-247. MR675187(84i:10023a)

[Sel56] Selberg, A.: Harmonic analysis and discontinuous groups in weakly symmetric Riemannian spaces with applications to Dirichlet series, J. Indian Math. Soc. (N.S.) 20 (1956), 47-87. MR0088511(19:531g)

[The05] The PARI Group, Bordeaux, PARI/GP, version 2.1.7, 2005, available from http://pari.math.u-bordeaux.fr/.

[Ven82] Venkov, A.B.: Spectral theory of automorphic functions, Proc. Steklov Inst. Math. (1982), no. 4(153), ix+163 pp. (1983), A translation of Trudy Mat. Inst. Steklov. 153 (1981). MR665585(85j:11060a)

[Wid41] Widder, D.V: The Laplace Transform, Princeton Mathematical Series, v. 6, Princeton University Press, Princeton, N. J., 1941. MR0005923 (3:232d)

[Zo82] Zograf, P.: Fuchsian groups and small eigenvalues of the Laplace operator, in Studies in Topology, IV, Zap. Nau cn. Sem. Leningrad. Otdel. Mat. Inst. Steklov. LOMI/ 122, 1982, in Russian; translated in Soviet Math. Dokl. 26 (1982), 24-29. MR661463 (84a:58090)

Department of Mathematics and Sciences, United States Merchant Marine Academy, 300 Steamboat Road, Kings Point, New York 11024

E-mail address: FriedmanJ@usmma.edu

E-mail address: joshua@math.sunysb.edu

E-mail address: CrownEagle@gmail.com

Department of Mathematics, The City College of New York, Convent Avenue at 138Th Street, New York, New York 10031

E-mail address: jjorgenson@mindspring.com

Institut für Mathematik, Humboldt-Universität ZU Berlin, Unter den Linden 6, D-10099 Berlin, Germany

E-mail address: kramer@math.hu-berlin.de 